\title{
Discrete element analysis of the punching behaviour of a secured drapery system: from laboratory characterization to idealized in situ conditions
}

\author{
Antonio Pol $^{1}$ (D) Fabio Gabrieli ${ }^{1}$ (D) $\cdot$ Lorenzo Brezzi $^{1}$ (D)
}

Received: 4 March 2020 / Accepted: 22 November 2020/Published online: 20 January 2021

(C) The Author(s) 2021

\begin{abstract}
In this work, the mechanical response of a steel wire mesh panel against a punching load is studied starting from laboratory test conditions and extending the results to field applications. Wire meshes anchored with bolts and steel plates are extensively used in rockfall protection and slope stabilization. Their performances are evaluated through laboratory tests, but the mechanical constraints, the geometry and the loading conditions may strongly differ from the in situ conditions leading to incorrect estimations of the strength of the mesh. In this work, the discrete element method is used to simulate a wire mesh. After validation of the numerical mesh model against experimental data, the punching behaviour of an anchored mesh panel is investigated in order to obtain a more realistic characterization of the mesh mechanical response in field conditions. The dimension of the punching element, its position, the anchor plate size and the anchor spacing are varied, providing analytical relationships able to predict the panel response in different loading conditions. Furthermore, the mesh panel aspect ratio is analysed showing the existence of an optimal value. The results of this study can provide useful information to practitioners for designing secured drapery systems, as well as for the assessment of their safety conditions.
\end{abstract}

Keywords Discrete element method (DEM) · Punch test · Rockfall protection · Rock slope stability $\cdot$ Secured drapery systems · Wire meshes

\section{Introduction}

Secured drapery systems are widely used as a countermeasure to rockfalls and shallow unstable phenomena along slopes. These systems are composed of a steel wire mesh, which is pinned to stable outcrops or to firm layers by means of tie rods or bolts and anchor plates (see Fig. 1). The stabilizing action that the mesh system can exert is strongly related to its deformation, which in turn is controlled by the load induced on it by the retained material [27] (e.g. unstable rock blocks, debris). This progressive activation of the mesh system retaining action highlights that secured draperies are characterized by passive behaviour [5, 9], and therefore, localized movements of the

\section{Antonio Pol}

antonio.pol@phd.unipd.it

1 Department of Civil, Environmental and Architectural Engineering, University of Padova, via Ognissanti 39, Padova, Italy retained material are expected in field applications. The knowledge of the force-displacement behaviour of the mesh system is therefore of fundamental importance for correct design; moreover, it permits one to evaluate system deformations in serviceability conditions. This aspect is particularly important in secured drapery interventions along railways and infrastructures.

Despite the large utilization of secured drapery systems, a lack of knowledge of their in-field mechanical behaviour exists and forecasting the latter in real applications still represents a challenging task. On one hand, the realization of real-scale experiments [4, 5] is complex and extremely costly. On the other hand, the laboratory tests that are currently used to evaluate the performance of the mesh do not correctly reproduce conditions in the field $[5,22,24,41]$. In the laboratory characterization, the mesh panel is generally fixed to a rigid frame along its outer boundary, instead in the field the mesh is only locally fixed by means of anchor plates. The standard tests therefore provide a mechanical characterization of the mesh that is 


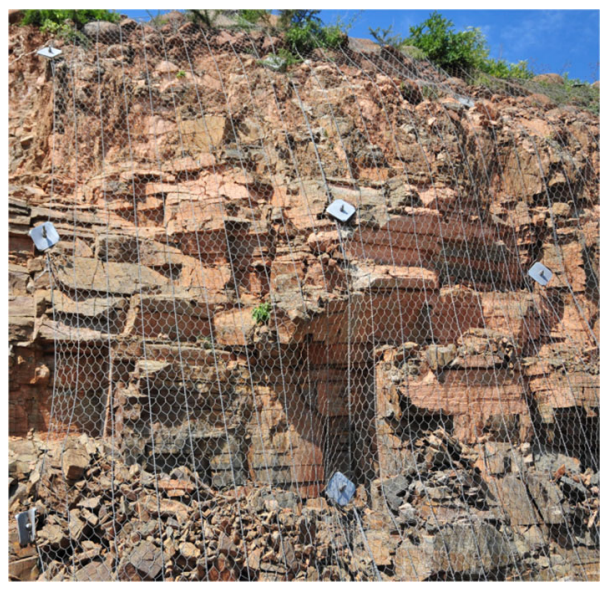

Fig. 1 Example of application of a secured drapery system (location: South Africa, courtesy of Dr. Giacchetti)

not representative of the in-field behaviour, which may lead to an overestimation of the mesh resistance.

The utilization of a numerical approach represents a valid alternative to expensive large-scale experimental tests, permitting one to easily modify the test configuration and providing an insight into the mechanical behaviour of the system. Several numerical works dealing with wire mesh structures, both considering the finite element method (FEM) $[13,18,19,23,25,26,40]$ and the discrete element method (DEM) [2, 6, 15, 32, 34, 37, 38, 42], are reported in the technical literature. In this work, the DEM is adopted because it can handle large deformations, multiple-contact problems and failures of mesh elements.

The main aim of this work is to extend the results of a standard laboratory characterization to a condition closer to the in-field one. In this perspective, the DEM is used to analyse the force-displacement response of an anchored mesh subjected to an out-of-plane loading condition. The common $8 \times 10$ hexagonal double-twisted steel wire mesh is considered, adopting a node-wire-based approach $[6,38]$ for the numerical representation of the mesh. The simulations are performed with the open-source code YADE [36]. Firstly, the mesh model is validated against experimental measurements (Sect. 3.2.1). Secondly, a large-scale anchored mesh system is analysed considering two different loading configurations (Sect. 4). Thirdly, the main variables of the problem (i.e. the punching element dimension and position, the size of the anchors and their spacing, the mesh panel geometry) have been modified in order to study their role on the mechanical performance of an anchored mesh panel (Sects. 4.1-4.5). The results obtained are used to define simple analytical relationships to account for the effect of the different variables of the problem. Finally, an application example is reported showing the use of the analytical relationships for extending the results of the standard laboratory characterization to field conditions (Sect. 4.6).

\section{Discrete element modelling of the wire mesh}

The DEM [16] represents an effective numerical tool for the analysis of multi-body mechanical problems that include contact, slipping and detachment between them in dynamic and quasi-static conditions. The first works adopting the DEM for the study of steel wire mesh structures were carried out by Hearn et al. [29], and subsequently by Nicot et al. [32, 33]. In the last two decades, the adoption of the DEM for analysing problems involving wire mesh structures has seen a large growth. More refined models have been implemented [7, 38] and applied to analyse the mechanical behaviour of wire meshes in laboratory conditions $[3,17,20,21,30]$ as well as in largescale applications. In particular, the dynamics of a single rock block impacting on wire mesh structures was studied in $[8,11,14]$. Moreover, the impact of a dry granular flow on a flexible barrier was analysed in [1, 2], while the impact of a debris flow (using a CFD-DEM coupled scheme) was considered in [31]. Only very recently, was the DEM applied to secured drapery systems [22, 34, 41, 42]. This is also the focus of the present work.

In a discrete element framework, a wire mesh is represented by a regular pattern of bodies connected by longrange remote interactions, or, more recently, directly joined to each other. The interaction between two connected mesh bodies (i.e. discrete elements) is governed by specific "contact" laws. Three different approaches are generally used to simulate a wire mesh: a cell-based approach (CB) $[15,32,33]$, a node-wire-based approach (NWB) $[6,7,35,37,38,41,42]$ and a cylinder-wire-based approach (CWB) [1, 2, 17, 21, 34].

In this work, the NWB approach is adopted. It permits reduction of the computational demand in comparison with the CWB approach and it does not introduce any approximation of the geometric pattern of the mesh elements (differently from the $\mathrm{CB}$ approach). In cases in which large bodies, in comparison with the mesh elementary cell, interact with the mesh, the NWB and the CWB approaches provide the same results in terms of mechanical response of the mesh [21]. In the NWB approach, each physical node of the mesh (i.e. intersection between two wires in the real mesh) is represented by a nodal spherical particle that concentrates the mass of the related wires. These nodal particles are connected by means of long-range interactions, thus representing the wires in the model (see Fig. 2a). This implies that the wires are not explicitly described in the numerical model; hence, contact with 


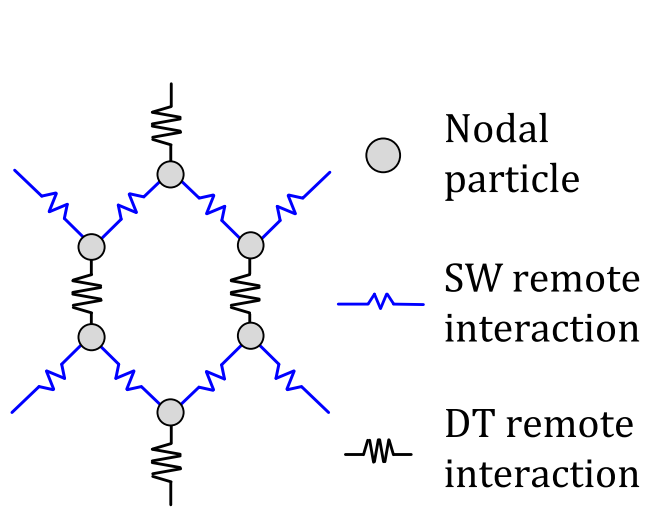

(a)

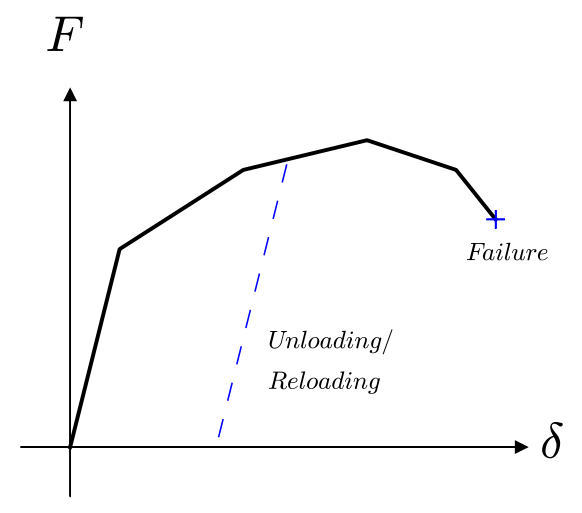

(b)

Fig. 2 a Graphical example of the mesh description with the NWB approach (adapted from [38]). b Graphical scheme of the EWM (adapted from [38])

external bodies is handled only at the level of the nodal particles. The interactions between the nodal particles are ruled by a user-defined piece-wise linear function (elementary wire model EWM) which describes the tensile force-displacement behaviour of the wires (compressive forces are neglected because of their low magnitude related to the effect of buckling [6]). The force-displacement relationship characterizing the EWM is derived from a stress-strain relationship (input data) on the basis of the defined wire diameter and the distance between two connected nodal particles. It should be noted that the wire diameter can be defined independently of the nodal particle diameter, thus permitting an optimization of the computational time [38]. An unloading/reloading condition is accounted for by using the same stiffness of the first branch of the force-displacement curve. The failure of a wire is considered (i.e. breakage of an interaction) by imposing a threshold value on the wire elongation. A schematic representation of the EWM is reported in Fig. 2b. The interaction at the contact point between a nodal particle and an external element is instead described by using the classic linear elastic-plastic contact law defined in [16]. The normal contact force $F_{n}$ is linearly related to the interpenetration $\delta$ (at the contact), while the tangential component $F_{t}$ is incrementally updated and is limited by a Coulomb's plastic condition. The normal and tangential contact forces are given by:

$$
\begin{aligned}
& F_{n}=k_{n} \delta \\
& \Delta F_{t}=k_{t} \dot{u} \mathrm{dt} \quad\left(\left|F_{t}\right| \leq\left|F_{n}\right| \tan \phi_{\mu}\right)
\end{aligned}
$$

where $k_{n}$ and $k_{t}$ are the normal and tangential contact stiffness, respectively, $\dot{u}_{t}$ the relative tangential velocity, $\mathrm{dt}$ the time step of the integration scheme and $\phi_{\mu}$ the friction angle at the contact between the two bodies.
The numerical simulations presented in this paper are performed with the open-source code YADE [36].

\section{Validation of the numerical model}

\subsection{Wire mesh characteristics}

In this work, the $8 \times 10$ hexagonal double-twisted wire mesh with a nominal wire diameter of $2.7 \mathrm{~mm}$ (Maccaferri) is considered. The periodic hexagonal cell is composed of two sub-elements: the single wires ( $\mathrm{SW}, \pm 45^{\circ}$ oriented) and the double-twisted wires (DT, aligned in parallel with respect to the machine manufacturing direction). The geometry of the hexagonal cell is described by three geometrical parameters: the characteristic lengths $a$ and $b$, both equal to $4 \mathrm{~cm}$, and the mesh opening size (mos) equal to $8 \mathrm{~cm}$ as shown in Fig. 3. In this work, the mechanical behaviour of the single and double-twisted wires is characterized by using the tensile stress-strain relationships presented in [38] and reported in Fig. 4. These are used for the definition of the force-displacement relationships (EWMs) that govern the interaction between two connected nodal particles in the numerical model (a different

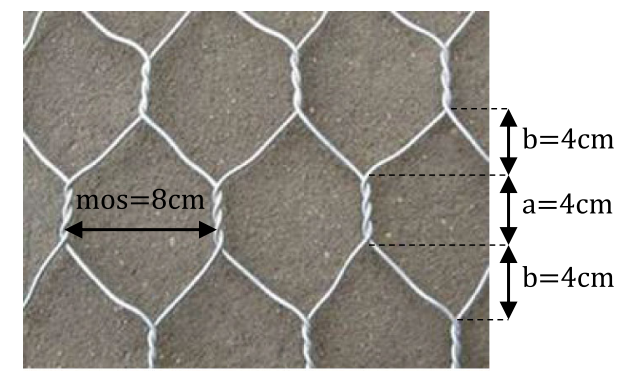

Fig. 3 View of the hexagonal double-twisted mesh with the indication of the geometrical parameters $a, b$ and mos 


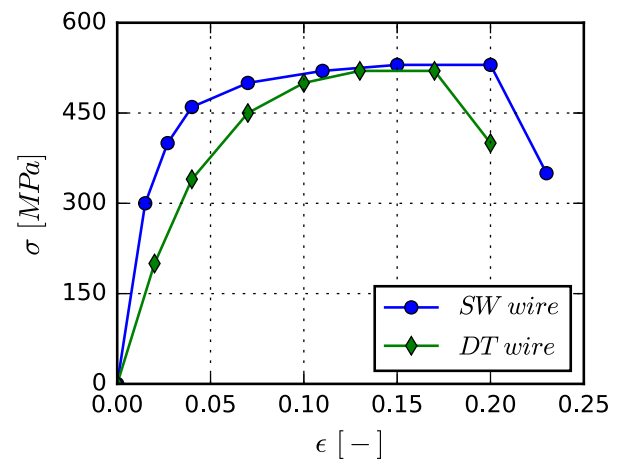

Fig. 4 Tensile stress-strain relationships of the single (SW) and double-twisted (DT) wires

EWM is used for describing the single and the doubletwisted wires).

The experimental results of a standard UNI punch test are used for the model validation. This test is widely utilized to investigate the response of a mesh panel against an out-of-plane loading condition. The standard UNI 11437 [39] prescribes the use of a spherical dome with a diameter $D=1.0 \mathrm{~m}$ and a curvature radius of $1.2 \mathrm{~m}$ (curvature radius of the dome edges equal to $0.05 \mathrm{~m}$ ) as punching element. Furthermore, a rectangular mesh panel with a nominal side of $3 \mathrm{~m}$ (tolerance of $20 \%$ ) must be utilized. In the experimental procedure, the mesh panel is fixed to an external rigid frame and the punching element is raised with a constant velocity $(\leq 0.01 \mathrm{~m} / \mathrm{s})$ until the complete failure of the mesh (i.e. the mesh is no longer able to contrast the punching element movement). During the test, the force acting on the punching element, as well as its displacement, are measured, providing as output a forcedisplacement relationship.

In the experimental test considered here, a mesh panel of dimensions $3 \mathrm{~m} \times 3 \mathrm{~m}$ is adopted. In addition to this standard punch test (i.e. $3 \mathrm{~m} \times 3 \mathrm{~m}$ mesh panel and dome diameter $1 \mathrm{~m}$ ), a "reduced" version, in which a $1 \mathrm{~m} \times 1 \mathrm{~m}$ mesh panel and a punching element of diameter $0.33 \mathrm{~m}$ are used, is performed in order to account for scale effects. A view of the test on the $3 \mathrm{~m} \times 3 \mathrm{~m}$ mesh panel is shown in Fig. 5b, while the experimental data are reported in Fig. 6.

\subsection{Numerical modelling of the punch test}

\subsubsection{Comparison to experimental results}

In order to validate the mesh numerical model, the experimental standard UNI punch test configuration described in Sect. 3.1 is numerically simulated. In the numerical model, the geometry of the experimental test is precisely reproduced. A mesh panel of dimensions $3 \mathrm{~m} \times 3 \mathrm{~m}$ is described by using 2890 nodal particles ( $\approx 322$ particles per $\mathrm{m}^{2}$ ). Following the strategy of [38], the nodal particles' diameter is set equal to four times that of real wires $\left(d_{n}=10.8 \mathrm{~mm}\right)$ : this entails a benefit in the computational time without influencing the mechanical response of the mesh (see Fig. 7a). The particles' density is thus adjusted in order to maintain the inertial proprieties of the mesh panel $\left(\approx 1.4 \mathrm{~kg} / \mathrm{m}^{2}\right.$ ). In order to reproduce the boundary conditions of the experimental test, the displacement of the particles belonging to the mesh outer boundary is fixed. Moreover, the rotational degrees of freedom of all the nodal particles are fixed (this permits frictional effects at the mesh-punch contact to be accounted for). The punching element geometry is precisely reproduced in the model by using 694 triangular elements. A sketch of the geometry adopted in the numerical punch test model is shown in Fig. 5a.

The numerical results are provided in terms of a forcedisplacement curve by recording the force acting on the punching element $(F)$ and its displacement $(\delta)$ during the test. The simulation is ended when the complete failure of the mesh is reached, namely when the tear in the mesh is sufficiently large to permit the punching element to pass through. In accordance with the experimental standard [39], the final point of the force-displacement curve refers to the maximum force value measured during the test. Finally, the time step is set equal to $\mathrm{dt}=3 \times 10^{-5} \mathrm{~s}$.

The contact parameters used for characterizing the mesh-punch contact are $k_{n}=6.5 \times 10^{5} \mathrm{~N} / \mathrm{m}, k_{t}=0.4 k_{n}$ and a $\phi_{u}=0$. It should be noted that these parameters have a slight effect on the numerical results (i.e. $F-\delta$ curve) as highlighted by the sensitivity analysis reported in Sect. 3.2.2. Furthermore, in order to reduce the computational time, the velocity imposed on the punching element in the numerical model is higher than the one used in the experimental procedure, and is equal to $v_{z}=0.1 \mathrm{~m} / \mathrm{s}$. This does not modify the mechanical response of the mesh panel as reported in Fig. $7 b$.

The numerical predictions are reported in Fig. 6a where they are compared with the experimental results. A good agreement is observed proving the effectiveness of the numerical approach. In addition, the "reduced" punch test configuration is simulated (i.e. $1 \mathrm{~m} \times 1 \mathrm{~m}$ mesh panel, punch diameter $D=0.33 \mathrm{~m})$. The same numerical parameters as before are adopted. The comparison between numerical and experimental results is in good agreement as shown in Fig. 6b. This provides a further confirmation of the validity of the mesh model; moreover, it highlights the capability of the model to reproduce scale effects. 


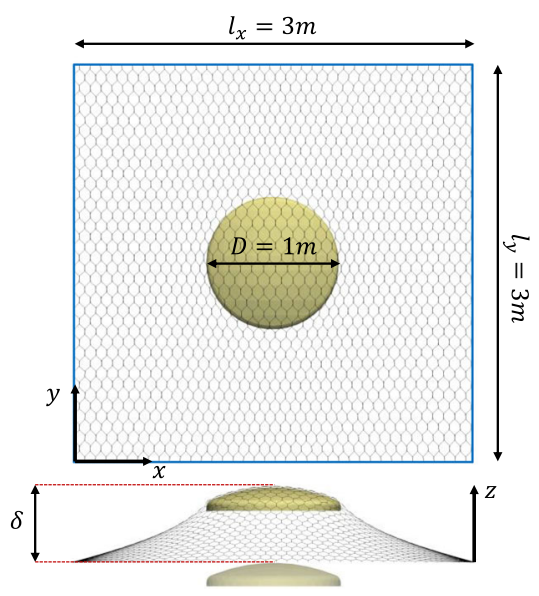

(a)

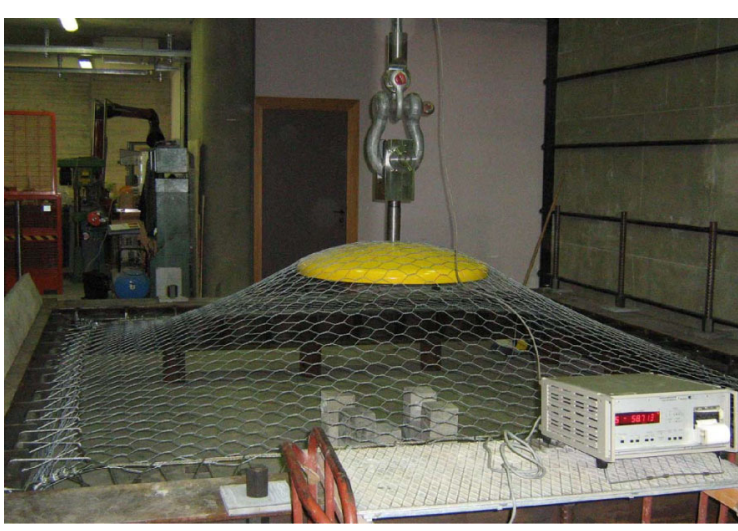

(b)

Fig. 5 a Sketch of the numerical model of the standard UNI punch test (blue solid lines represent the fixed mesh boundary). b View of the experimental standard UNI punch test (test conducted by Maccaferri) (color figure online)

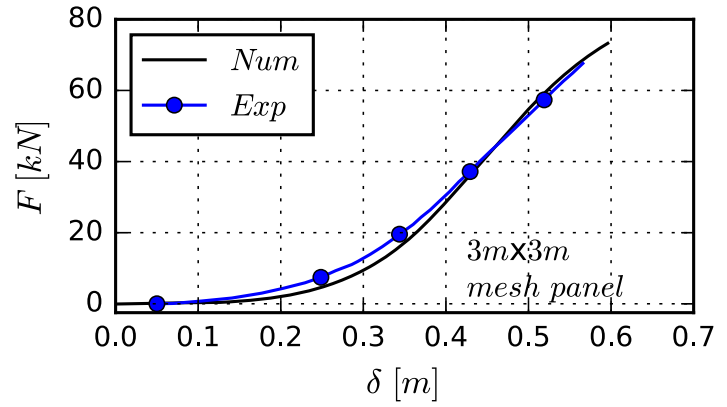

(a)

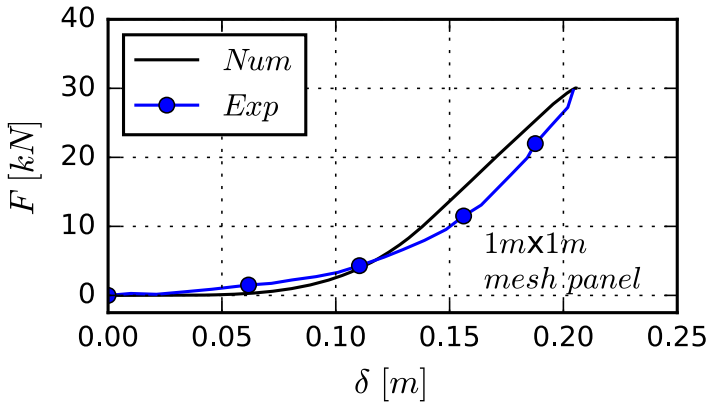

(b)

Fig. 6 Comparison between numerical and experimental punch test results for: a the standard UNI test on a $3 \mathrm{~m} \times 3 \mathrm{~m}$ mesh panel $(D=1.0 \mathrm{~m})$ and $\mathbf{b}$ the "reduced" test on a $1 \mathrm{~m} \times 1 \mathrm{~m}$ mesh panel $(D=0.33 \mathrm{~m})$

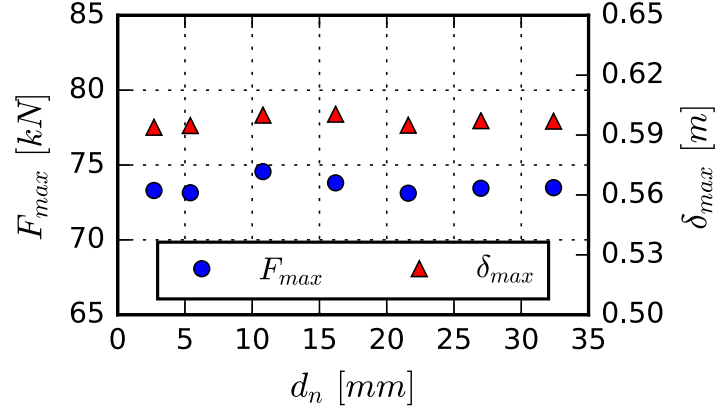

(a)

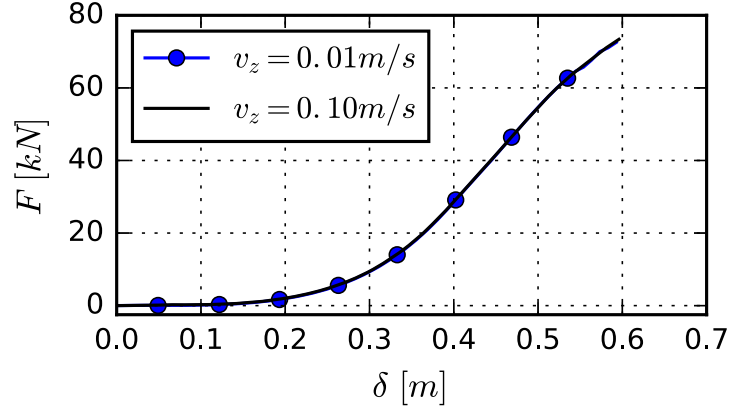

(b)

Fig. 7 Effect of the nodal particle diameter $d_{n}$ in terms of force $F_{\max }$ and deflection $\delta_{\max }$ values at failure. $\mathbf{b}$ Effect of the punching element lifting velocity $v_{z}$ on the mechanical response of the mesh

\subsubsection{Sensitivity analysis of the mesh-punch contact parameters}

The influence of the normal contact stiffness is analysed varying its value in the range $6.5 \times 10^{3} \mathrm{~N} / \mathrm{m} \leq k_{n} \leq 2.6 \times 10^{7} \mathrm{~N} / \mathrm{m}$. A variation of $k_{n}$ induces a variation of $k_{t}$ as well; however, since the meshpunch contact is assumed frictionless (i.e. $\phi_{\mu}=0$ ), this does not influence the tangential behaviour at the meshpunch contact. The numerical results (Fig. 8a) show that for $k_{n} \geq 1 \times 10^{5}$, this parameter does not significantly influence the mechanical response of the mesh (differences 


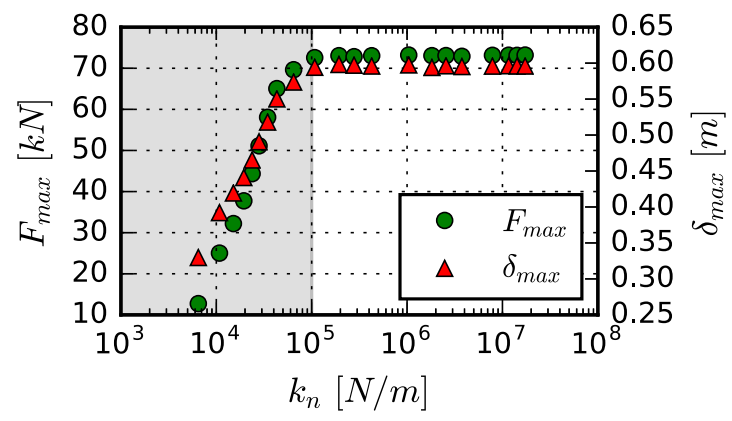

(a)

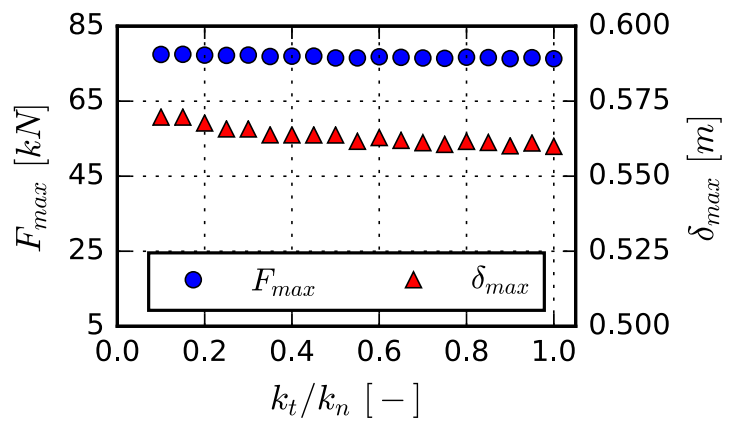

(b)

Fig. 8 Effect of the mesh-punch a normal contact stiffness $k_{n}$ and $\mathbf{b}$ tangential contact stiffness $k_{t}$ in terms of force $F_{\max }$ and deflection $\delta_{\max }$ values at failure

in terms of force and displacement values at failure are less than $1 \%$.). For lower values of $k_{n}$, the interpenetration between the mesh particles and the facet elements, at a certain point of the simulation (depending on the $k_{n}$ value), becomes too large compromising the correctness of the simulation, namely the punching element passes through the mesh before the failure of the latter. However, before this moment the mechanical response of the mesh is the same independently of the value $k_{n}$ (i.e. $F-\delta$ curves are superimposed).

For the analysis of the effect of the tangential contact stiffness $k_{t}$, an extremely high value of the contact friction angle $\left(\phi_{\mu}=89^{\circ}\right)$ is used in order to maximize the possible differences due to tangential effects at the mesh-punch contact. The value of $k_{t}$ is varied in a wide range $0.1 k_{n} \leq k_{t} \leq 1.0 k_{n} \quad\left(k_{n}=6.5 \times 10^{5} \mathrm{~N} / \mathrm{m}\right)$. The results reported in Fig. $8 \mathrm{~b}$ show that the influence of $k_{t}$ is also negligible (differences in terms of force and displacement values at failure are less than $1.3 \%$.).

Finally, the mesh-punch contact friction angle $\phi_{\mu}$ is varied from a frictionless case up to the extremely high frictional condition $\phi_{\mu}=89^{\circ} \quad\left(k_{n}=6.5 \times 10^{5} \mathrm{~N} / \mathrm{m}\right.$, $\left.k_{t}=0.4 k_{n}\right)$. The numerical results obtained for $\phi_{\mu}=0^{\circ}$, $\phi_{\mu}=10^{\circ}, \quad \phi_{\mu}=89^{\circ}$ are compared in Fig. 9. The

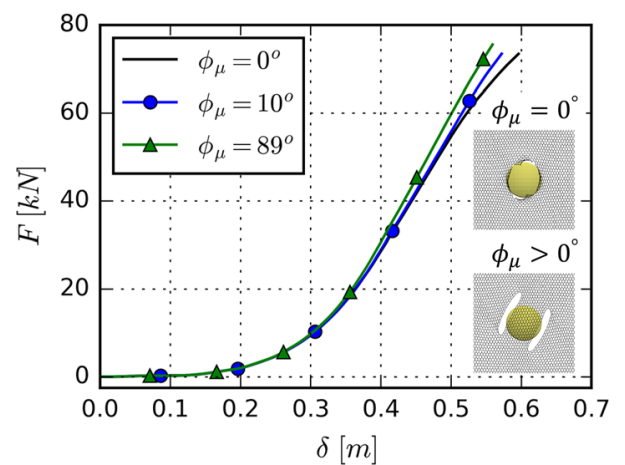

Fig. 9 Effect of the mesh-punch contact friction angle in terms of force-displacement curve mechanical response in the initial part of the force-displacement curve (i.e. $\delta \leq 0.35 \mathrm{~m}$ ) is the same for all three cases, then the curves diverge very slightly. Considering the two extreme cases (i.e. $\phi_{\mu}=0^{\circ}$ and $\phi_{\mu}=89^{\circ}$ ), the difference is approximately equal to $4 \%$ and $6 \%$ concerning the force and the deflection values at failure, respectively. This shows the slight influence of the contact friction angle on the mesh response. This fact is ascribable to the particular testing conditions: on one hand, the rounded shape of the punch limits the mesh sliding on contact with the punching element, on the other hand, the loading direction (i.e. normally oriented to the mesh plane) entails that tangential forces have a marginal effect on the mesh mechanical behaviour.

From a numerical perspective, it is interesting to note that the failure modality is different moving from a frictionless to a frictional case as shown in Fig. 9. In the former, the mesh starts to break just in the centre of the panel, while the failure occurs near the edges of the punching element for a frictional case. In the experimental test, the failure starts approximately from the centre of the mesh panel and propagates parallel to the double-twisted wires orientation. This condition is similar to the one obtained adopting $\phi_{\mu}=0$. Having said that, given the low influence of $\phi_{\mu}$ on the mesh response and the lack of knowledge of a precise value of the mesh-punch friction coefficient, a frictionless mesh-punch contact is adopted representing the most critical condition for the mesh (i.e. $\phi_{\mu}=0 \mathrm{~min}$ imizes the force value at failure).

In light of the above reported results, it is evident that the mesh-punch contact parameters play a negligible role in the problem under consideration. Indeed, the problem is controlled by the tensile behaviour of the mesh, which is ruled by the contact laws governing the mesh particles' interactions (i.e. EWMs). The EWMs are defined from stress-strain tensile relationships obtained from the experimental tensile test. In this sense, since deterministic 
EWMs are used here, a more detailed calibration of the numerical parameters is not needed.

\subsubsection{Effect of the size of the punching element}

The standard UNI punch test is widely used for the characterization of the punching resistance of a wire mesh $[5,20,41]$; however, other configurations are adopted in practice $[10,12]$. The difference between the test procedures, which is mainly related to the punching element dimension, makes direct comparison between the experimental results in the technical literature difficult. In order to evaluate the effect related to a variation of the punching element dimension, the latter has been varied ranging from $D=0.1 \mathrm{~m}$ to $D=2.5 \mathrm{~m}$ with an incremental step of $0.1 \mathrm{~m}$. In all the simulations, a $3 \mathrm{~m} \times 3 \mathrm{~m}$ panel is considered and the contact parameters are the same as in Sect. 3.2.1 $\left(k_{n}=6.5 \times 10^{5} \mathrm{~N} / \mathrm{m}, k_{t}=0.4 k_{n}, \phi_{\mu}=0^{\circ}\right)$.

In Fig. 10, the mesh mechanical response, obtained for five values of $D$ (i.e. $0.5 \mathrm{~m}, 1.0 \mathrm{~m}, 1.5 \mathrm{~m}, 2.0 \mathrm{~m}, 2.5 \mathrm{~m}$ ), is reported. The trend of the values of the force and the deflection at failure, in the entire range of variation of $D$, is instead reported in Fig. 11a, b, respectively. In Fig. 11, the values are normalized on the ones obtained in the standard UNI test $\left(F^{U}=73.3 \mathrm{kN}, \delta^{U}=0.60 \mathrm{~m}\right)$ to facilitate the comparison.

The numerical results show that the mesh puncturing resistance $F_{\max }$ linearly scales with the punching element dimension $D$ (see Fig. 11a). For example, increasing the UNI punching element diameter by a factor of 2 (i.e. $D=2.0 \mathrm{~m}$ ) results in twice the $F_{\max }$ value with respect to the UNI condition $\left(F_{\max }(D=2.0 \mathrm{~m})=146.3 \mathrm{kN}\right)$. The force sustained by the mesh panel can be viewed as an integral of the tensile forces supported by the wires along the punching element boundary. A larger punching element intercepts a higher number of wires (this linearly scales with the punch diameter $D$ ) and therefore determines an

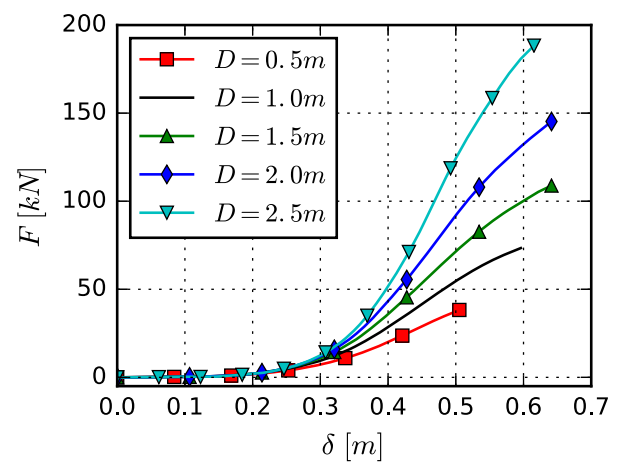

Fig. 10 Effect of the punching element dimension in terms of forcedisplacement curve increase in the maximum force supportable by the mesh panel. The relationship between the maximum force and the punching element dimension $\left(D^{U}=1.0 \mathrm{~m}\right)$ is well represented by the following expression (linear least squares fit):

$F_{\max }=\frac{D}{D^{U}} F^{U}$

Equation 3 accounts for the effect of the punching element dimension on the puncturing resistance of a mesh panel. This permits the results obtained from different test configurations (i.e. different dimensions of the punching device) to be extended to a "common" dimension of the punching element, thus allowing a first comparison between the different data reported in the technical literature. It should be noted, that possible effects deriving from the shape of the punching device, as well as from its orientation with respect to the mesh wires (wire meshes are generally characterized by anisotropic behaviour in the two principal directions) were not considered in the definition of Eq. 3.

The value of the deflection at failure $\delta_{\max }$ increases as a linear function up to $D=1.2 \mathrm{~m}$, then it reaches a plateau as reported in Fig. 11b. The relationship between the deflection at failure and the punching element dimension $\left(D^{U}=1.0 \mathrm{~m}\right)$ is well represented by the following expression (linear least squares fit):

$$
\left\{\begin{array}{cc}
\delta_{\max }=\left(0.34 \frac{D}{D^{U}}+0.66\right) \delta^{U} & \text { if } \frac{D}{l_{y}}<0.4 \\
\delta_{\max }=1.07 \delta^{U} & \text { if } \frac{D}{l_{y}} \geq 0.4
\end{array}\right.
$$

The specific trend of $\delta_{\max }$ proves that the finite size of the mesh panel acts as a limiting factor. In this perspective, the emergence of the plateau of $\delta_{\max }$ should be related to the ratio $D / l_{y}$. In particular, the ratio between the size of the punching element and the mesh side length $l_{y}$ affects the strain distribution in the wires. For $D / l_{y} \geq 0.4$, some of the wires along the mesh outer boundary experience a strong tensile strain (i.e. $\epsilon \geq 0.05$ ) and start to experience plastic deformation as reported in Fig. 12. From this moment, the mesh panel cannot redistribute the load coming from the punching element and a further displacement leads to a localization of strains in the centre of the mesh panel triggering the failure of the latter. As for the out-of-plane stiffness of the mesh panel, it increases when increasing the dimension of the punching element (see Fig. 10). In fact, increasing $D$ leads to a better distribution of tensile stress on the panel, which entails a stiffer response of the mesh panel. 


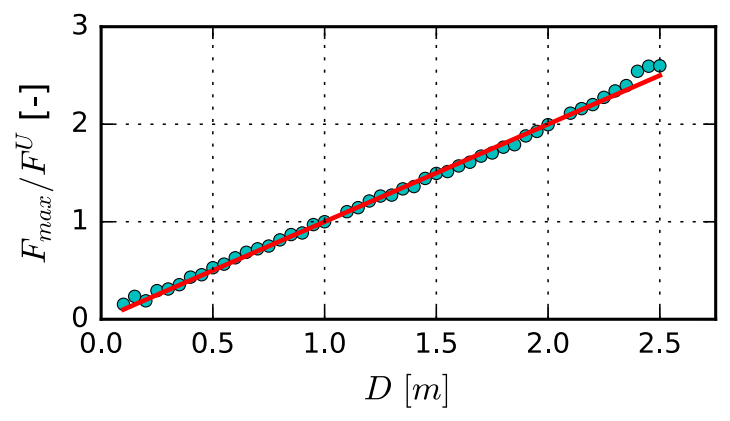

(a)

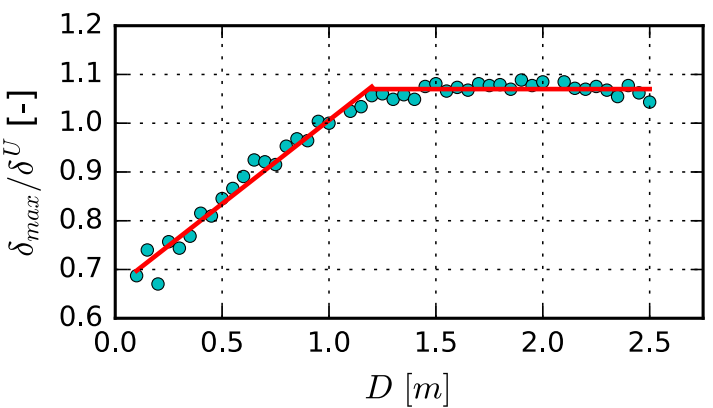

(b)

Fig. 11 Effect of the punching element dimension in terms of: a force $F_{\max }$ (fit Eq. 3) and $\mathbf{b}$ deflection $\delta_{\max }$ (fit Eq. 4) values at failure

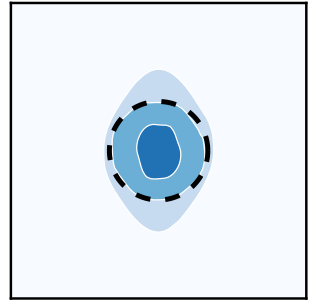

(a)

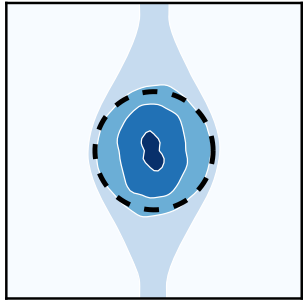

(b)

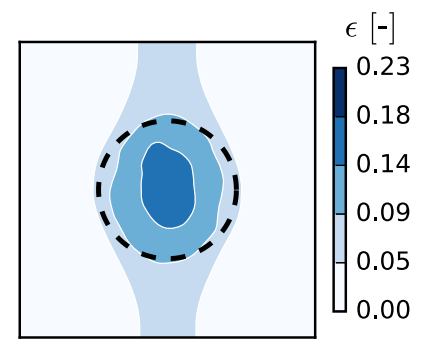

(c)

Fig. 12 Strain contour plot of the wires prior to failure for: $\mathbf{a} D=1.0 \mathrm{~m}, \mathbf{b} D=1.2 \mathrm{~m}, \mathbf{c} D=1.4 \mathrm{~m}$. The contour plots are obtained from a linear interpolation of the strain acting on the wires on a regular grid with a spacing of $4 \mathrm{~cm}$

\section{Mechanical behaviour of an anchored mesh panel}

The standard UNI punch test procedure is used as an informative test for the design of secured drapery interventions. Nevertheless, the boundary conditions imposed on the mesh panel (i.e. fixed outer boundary), substantially differ from the ones characterizing field applications in which the mesh is only locally anchored. This difference determines an incorrect characterization of the mesh mechanical behaviour, which, if not duly considered, may negatively reflect on the performance of the mesh system. While the implementation of an "in situ" test set-up may be difficult to achieve (an example of large-scale test can be found in [5]), the use of a numerical approach allows this problem to be overcome [22, 37, 41].

In this work, in order to move towards typical field conditions two large-scale configurations were simulated in the first phase. In both configurations, the mesh has dimensions $12 \mathrm{~m} \times 12 \mathrm{~m}$ (45152 nodal particles) and is subdivided into 9 square mesh panels $(3 \mathrm{~m} \times 3 \mathrm{~m}$ each); the distance between the borders of the lateral mesh panels and the outer boundary of the mesh system was set equal to $1.5 \mathrm{~m}$ to limit possible boundary effects (see Fig. 13). The presence of the anchor plates is simulated by fixing the degrees of freedom of the nodal particles ideally intercepted by the anchor plates. Square anchor plates of side $d_{p}=0.32 \mathrm{~m}$ were considered in the model, which represents a standard plate dimension in practice. The loading condition is simulated by using the same punching element (or several punching elements) and the same contact parameters adopted in Sect. 3.2.1. The punching element is moved with a constant velocity $\left(v_{z}=0.1 \mathrm{~m} / \mathrm{s}\right)$ in a direction perpendicular to the mesh plane until the complete failure of the mesh. In this case (i.e. anchored mesh panels), the failure condition is determined by the tear of the mesh at the level of the anchor plates. In the first configuration (Test A), a single punching element is considered, acting in the centre of the mesh system as shown in Fig. 13a. This condition ideally simulates the load caused by a single unstable block similarly to what was considered in [41]. In the second configuration (Test B), a punching element is considered acting on each mesh panel (i.e. 9 punching elements are simulated) as shown in Fig. 13b. This condition ideally simulates the restrictive case in which each mesh panel has to support a single block and cannot share the external load with neighbouring panels, as instead happens in Test A. In both configurations, the output is represented by the force-displacement curve referred to the punching element acting on the central mesh panel (in Test $B$ differences in terms of maximum force are lower than $10 \%$ between the different panels); the final point of the force-displacement curve refers to the maximum force value measured during the test. The mechanical 


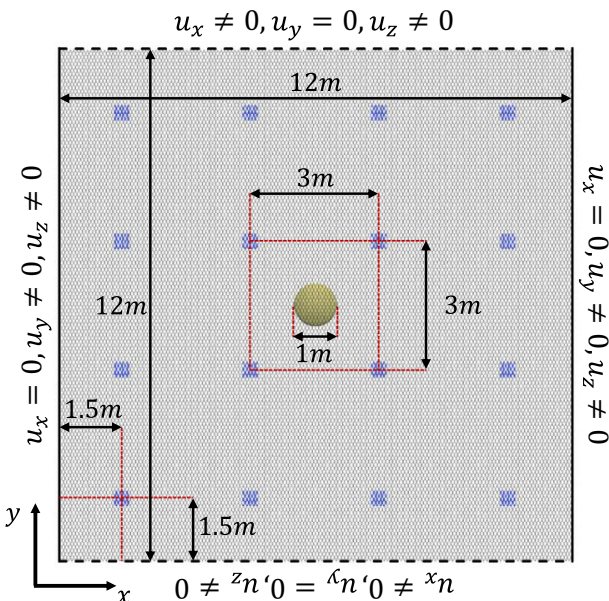

(a)

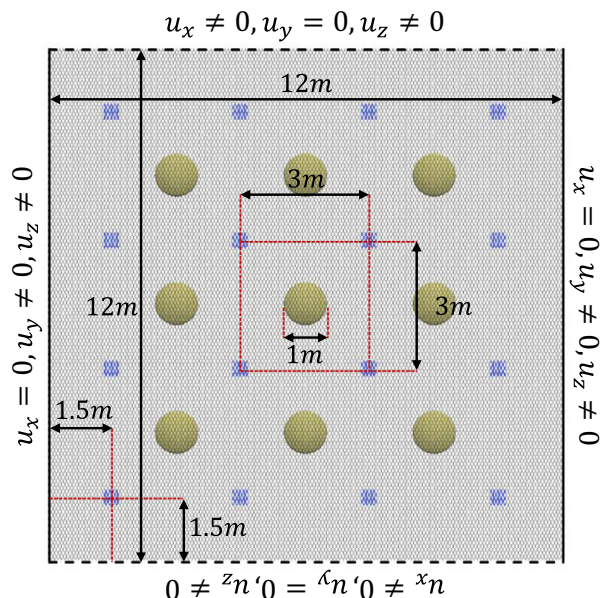

(b)

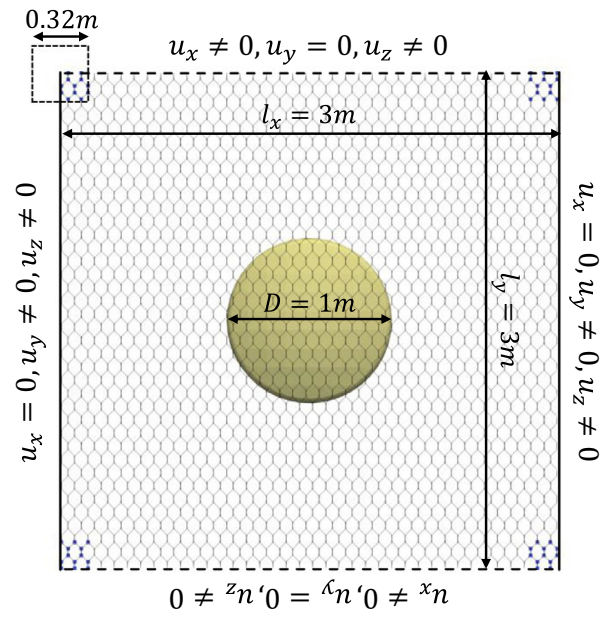

(c)

Fig. 13 a Test configuration Test $A$, b test configuration Test B, $\mathbf{c}$ test configuration Test B1 (the nodal particles ideally intercepted by the anchor plates are represented in blue) (color figure online)

response obtained in Test $A$ and Test $B$ is reported in Fig. 14. In the former configuration a higher localized

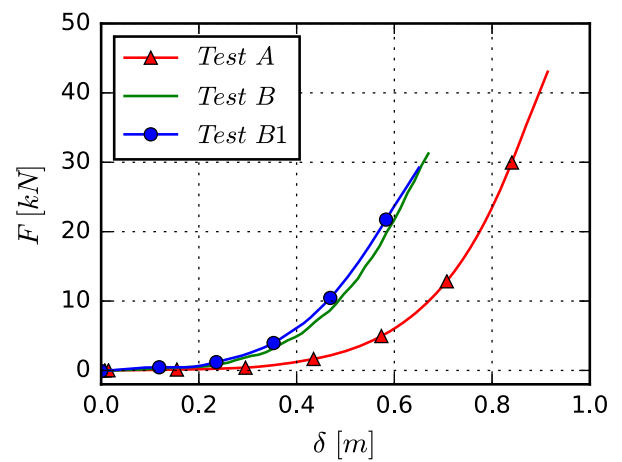

Fig. 14 Comparison of the force-displacement curves obtained from the different test configurations (Test A, Test B, Test B1) force can be sustained by the mesh system $\left(F_{\max }=43.4 \mathrm{kN}\right.$ in Test $A, F_{\max }=31.3 \mathrm{kN}$ in Test $\left.B\right)$, because the load can be shared between the different panels (i.e. the central panel can partially transmit the external load to adjacent panels). Even if a condition similar to the Test $B$ configuration is unlikely in practice, it provides a lower bound estimation of the puncturing resistance of an anchored mesh panel. From the perspective of characterizing the mesh resistance to be used in the design phase of secured drapery systems, the adoption of Test $B$ configuration is more conservative.

Finally, the Test $B$ configuration is schematized by using a "periodic" single panel test configuration (Test Bl). In this a quarter of a square anchor plate of side length $d_{p}$ is considered at the panel corners and periodic boundary conditions are imposed on the panel edges (i.e. the 
Table 1 Geometrical parameters and numerical mesh-punch contact parameters

\begin{tabular}{ll}
\hline Panel size $l_{x}$ & $3.00 \mathrm{~m}$ \\
Panel size $l_{y}$ & $3.00 \mathrm{~m}$ \\
Anchor plate side $d_{p}$ & $0.32 \mathrm{~m}$ \\
Punching element diameter $D$ & $1.00 \mathrm{~m}$ \\
Punching element position $(x, y)$ & $1.5 \mathrm{~m}, 1.5 \mathrm{~m}$ \\
Normal contact stiffness $k_{n}$ & $6.5 \times 10^{5} \mathrm{~N} / \mathrm{m}$ \\
Tangential contact stiffness $k_{t}$ & $0.4 k_{n}$ \\
Contact friction angle $\phi_{\mu}$ & $0.0^{\circ}$ \\
\hline
\end{tabular}

displacement along the direction parallel to the mesh side is fixed). For the sake of clarity, a sketch of the model of the Test Bl configuration is reported in Fig. 13c. This approximation permits a significant reduction of the computational cost of the simulation, and entails a negligible effect on the mechanical response of the mesh as observable comparing the force-displacement curves reported in Fig. 14. Therefore, the "periodic" single panel configuration is adopted in the following in order to analyse the mechanical response of an anchored mesh panel. The numerical and geometrical parameters characterizing the model are summarized in Table 1 .

In order to quantify the difference in terms of mechanical response of the mesh panel, moving from the laboratory to field conditions (i.e. anchored mesh panel), the force-displacement curve obtained from the standard UNI punch test is compared with the one obtained in the Test BI configuration as reported in Fig. 15a. A significant reduction of the maximum sustainable force is observed when considering more realistic boundary conditions: $F^{U}=73.3$ $\mathrm{kN}$ in the standard UNI configuration, $F_{a}^{*}=29.1 \mathrm{kN}$ in the Test Bl configuration. This is due to the lower number of wires that can sustain an out-of-plane force (i.e. fixed nodal particles in the model). In the standard UNI punch test all the wires converging to the panel outer boundary are able to sustain an out-of-plane load; considering an anchored panel (i.e. Test B1), the number of wires that can sustain such a kind of loading condition is significantly lower (i.e. only the wires intercepted by the anchor plates). The failure modality is also different in the two cases: in the standard UNI punch test the failure is observed in correspondence with the punching element (see Fig. 15b), while it occurs in proximity to the anchor plates considering the anchored mesh panel (see Fig. 15c). The ratio between the maximum force obtained in standard UNI conditions and the one obtained considering an anchored mesh panel $\left(\alpha_{F}=F_{a}^{*} / F^{U} \approx 0.40\right)$ can be used, to a first approximation, in order to estimate the reduction of the panel's ultimate strength moving from laboratory to field conditions. The same reasoning can be applied for the maximum deflection $\left(\alpha_{\delta}=\delta_{a}^{*} / \delta^{U} \approx 1.08\right)$. However, these results are strongly

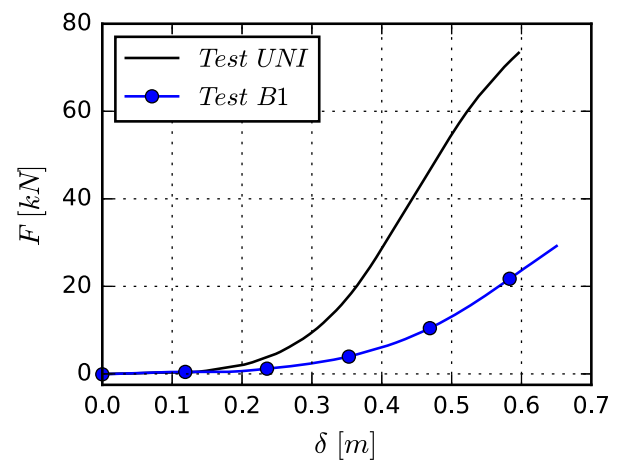

(a)

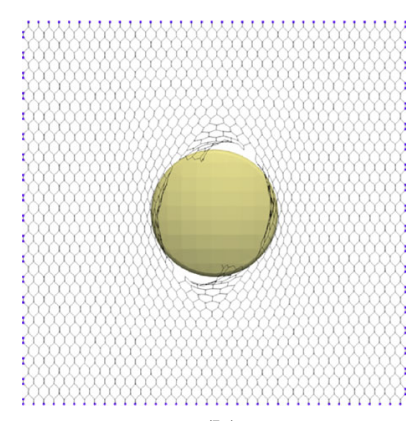

(b)

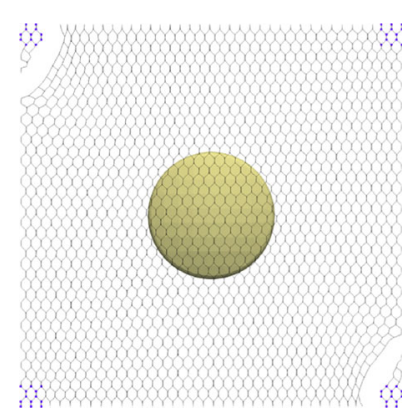

(c)
Fig. 15 a Comparison of force-displacement curve obtained from the standard UNI punch test and the configuration Test B1. Failure modes observed for $\mathbf{b}$ the standard UNI punch test and $\mathbf{c}$ the configuration Test B1

related to the geometry of the problem (e.g. anchor size and spacing, punching element size) and the mesh mechanical properties, therefore, should be carefully considered.

Once the in situ punch test configuration is defined (i.e. Test B1), a parametric analysis is performed in order to extend the results of the standard mesh characterization (i.e. UNI punch test) to more general field conditions. The influence of the size and position of the punching element as well as the role of anchor plate dimension and spacing are discussed in the following. Finally, the dependency of the mechanical response on the aspect ratio of the mesh panel (i.e. $A R=l_{x} / l_{y}$ ) is investigated. The numerical simulations are characterized by the parameters reported in Table 1, with the only exception of the parameter under investigation (the range of variation will be explicitly specified in the related paragraph). For facilitating the comparison, all the results are normalized on the values of the force $F_{a}^{*}=29.1 \mathrm{kN}$ and of the deflection $\delta_{a}^{*}=0.65 \mathrm{~m}$ at failure, obtained in the reference test (i.e. Test B1, parameters are reported in Table 1).

\subsection{Effect of the size of the punching element}

In the design phase of a secured drapery system, the expected dimension and sharpness of the unstable blocks 
should be carefully considered to optimize the system's mechanical performance. The knowledge of how the geometry of the retained block influences the mechanical response of the mesh panel is therefore of great importance. In this perspective, dimension $D$ of the punching element is varied ranging from $0.1 \mathrm{~m}$ to $2.5 \mathrm{~m}$ with an incremental step of $0.1 \mathrm{~m}$. The other parameters are the same as those in the reference test reported in Table 1. A variation of the dimension of the punching element permits consideration, in addition to scale effects, also of the impact of block sharpness on the mesh mechanical response. The main effect of block angularity is to vary the contact surface between the block and the mesh, thus a reduction of the punching element dimension may represent a simple but effective way to consider the reduction of the contact surface associated with the increment of block angularity. In other words, the sharper the assumed block, the lower the punching element dimension considered in the model.

The mechanical response of the mesh panel, obtained for five different values of $D(0.1 \mathrm{~m}, 0.2 \mathrm{~m}, 0.4 \mathrm{~m}, 1.0 \mathrm{~m}$, $2.0 \mathrm{~m}$ ), is reported in Fig. 16. The trend of the values of the force and the deflection at failure, in the entire range of variation of $D$, is instead reported in Fig. 17a, b, respectively. Furthermore, an estimation of the out-of-plane stiffness of the mesh panel is provided by means of the parameter $k_{75}$. This is defined as the stiffness of the $F-\delta$ curve at $75 \%$ of the maximum force. The trend of the stiffness parameter $k_{75}$ is reported in Fig. 17c $\left(k_{75}^{*}=105\right.$ $\mathrm{kN} / \mathrm{m})$.

The punching resistance of the mesh panel (i.e. $F_{\max }$ ) considerably increases when increasing the punching element dimension for $D \leq 0.40 \mathrm{~m}$. After this threshold, the increase in $F_{\max }$ with $D$ is greatly reduced as shown in Fig. 17a. Such a change of behaviour is related to the variation of the failure modality of the mesh panel. In the former case (i.e. $D \leq 0.40 \mathrm{~m}$ ), the mesh failure starts at the centre of the panel, namely in correspondence to the

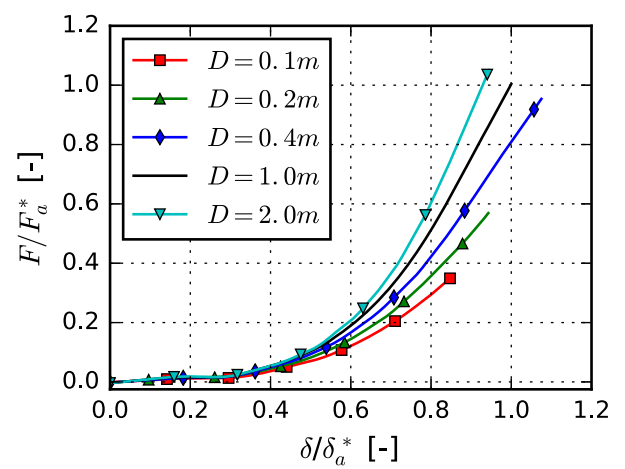

Fig. 16 Effect of the punching element dimension in terms of forcedisplacement curve punching element. In the latter case (i.e. $D>0.40 \mathrm{~m}$ ), instead, the failure moves close to the anchor plates. The two failure modalities are shown in Fig. 18. The variation of failure modality is observed for a threshold value of $D$ that is very close to the anchor plate dimension $\left(D=0.40 \mathrm{~m}\right.$ and $\left.d_{p}=0.32 \mathrm{~m}\right)$. The maximum force of an anchored mesh panel is limited by the available resistance at the mesh-plate connection, which can be considered a function of the number of wires intercepted by an anchor plate. In this perspective, when considering a punching element characterized by a dimension lower than the anchor plate dimension, the former intercepts a lower number of wires, and thus the mesh fails in correspondence with the punching element. In the opposite case (i.e. $D \gtrsim d_{p}$ ), the larger interacting surface between the mesh and the punch permits avoidance of a premature failure, and the mesh fails at the mesh-plate connection when the maximum available resistance of the latter is overcome. In this case, the anchor plate can be seen as the "new punching element". The two observed failure modalities can be characterized on the basis of where the local punching mechanism takes place. In this sense, it is possible to define a "block-punching" failure mechanism when $D \lesssim d_{p}$ and an "anchor-punching" failure mechanism when $D \gtrsim d_{p}$. Finally, the slight increment of the maximum force observed when increasing the punching element dimension over the threshold values $D>0.40 \mathrm{~m}$ may be attributable to a better redistribution of the forces inside the mesh panel.

The effect of the two different failure mechanisms is also observable with regard to the trend of the deflection at failure $\delta_{\max }$ as reported in Fig. $17 \mathrm{~b}$. An increase in $\delta_{\max }$ with $D$ is observed if the mesh failure is characterized by the "block-punching" mechanism. In fact, under this condition an increase in $D$ permits a delay of the failure of the mesh panel related to the puncturing at the punch-mesh contact. Conversely, if the mesh failure is characterized by the "anchor-punching" mechanism, a reduction of $\delta_{\max }$ when increasing $D$ is observed. In this case, a larger punching element determines a prompter response of the mesh, the distance between the punching element edges and the anchor plates being lower, thus determining a reduction of the mesh panel deflection.

Finally, it is interesting to analyse the influence of the punching element dimension on the mesh panel out-ofplane stiffness (represented by using the stiffness parameter $k_{75}$ ). The numerical data (Fig. $17 \mathrm{c}$ ), show a linear increase in the stiffness parameter $k_{75}$ with the punching element dimension $D$, and no significant differences are observed moving from one to the other failure mechanism. The increase in the stiffness parameter $k_{75}$ with the punching element dimension is therefore related to the greater 


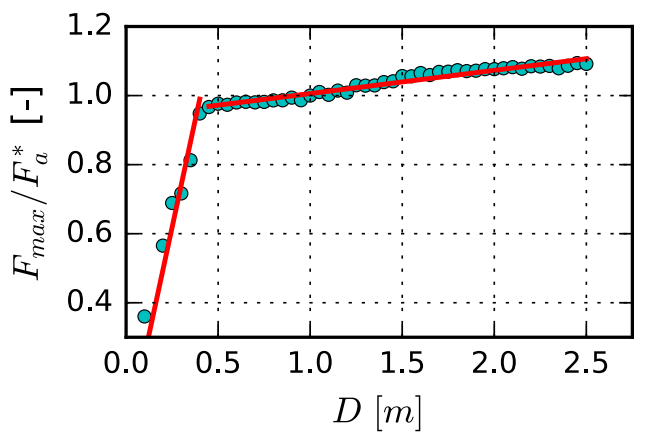

(a)

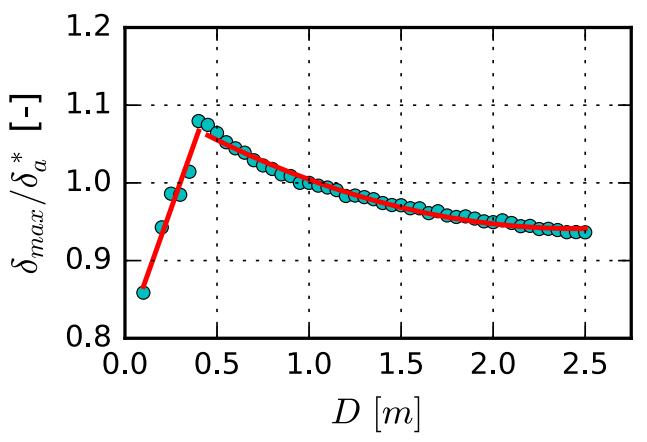

(b)

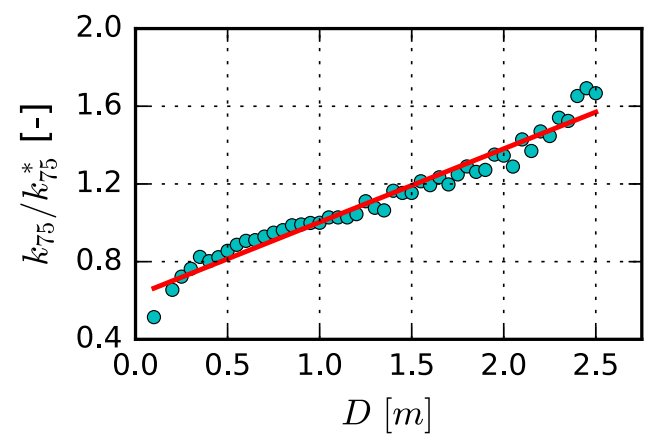

(c)

Fig. 17 Effect of the punching element dimension in terms of: a force $F_{\max }$ (fit Eq. 5), b deflection $\delta_{\max }$ (fit Eq. 6) values at failure and $\mathbf{c}$ stiffness parameter $k_{75}$ (fit Eq. 7)

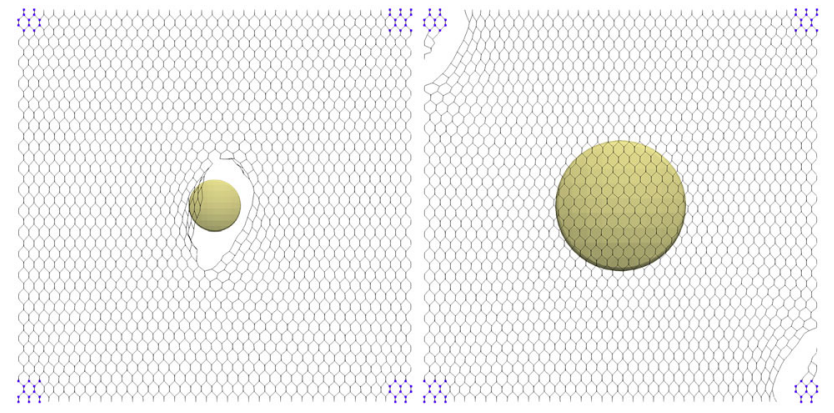

(a)

$$
\begin{aligned}
& \left\{\begin{array}{cc}
F_{\max }=2.47 \frac{D}{D^{*}} F_{a}^{*} & \text { if } D \lesssim d_{p} \\
F_{\max }=\left(0.06 \frac{D}{D^{*}}+0.94\right) F_{a}^{*} & \text { if } D \gtrsim d_{p}
\end{array}\right. \\
& \left\{\begin{array}{cc}
\delta_{\max }=\left(0.72 \frac{D}{D^{*}}+0.78\right) \delta_{a}^{*} & \text { if } D \lesssim d_{p} \\
\delta_{\max }=\left[0.03\left(\frac{D}{D^{*}}\right)^{2}-0.16 \frac{D}{D^{*}}+1.13\right] \delta_{a}^{*} & \text { if } D \gtrsim d_{p}
\end{array}\right.
\end{aligned}
$$

$k_{75}=\left(0.38 \frac{D}{D^{*}}+0.62\right) k_{75}^{*}$ punch dimension of a $D=0.40 \mathrm{~m}, \mathbf{b} D=1.0 \mathrm{~m}$ (reference case). The nodal particles ideally intercepted by the anchor plates are represented in blue (color figure online)

proximity of the punching element edges to the anchor plates.

From the numerical data, the relationships permitting estimation of the effect of a variation of the punching element size $D$ on the force (linear least squares fit) and deflection (second-order polynomial least squares fit) values at failure and on the stiffness parameter $k_{75}$ (linear least squares fit) can be derived (the punch dimension is related to the reference value $D^{*}=1.0 \mathrm{~m}$ ). They are given by:

\subsection{Effect of the position of the punching element}

The position of a possible unstable block on the mesh panel, or more precisely, the relative position between the block and the anchors, can somehow be controlled during the installation of a secured drapery system. Therefore, it may be interesting to investigate its effect on the mechanical response of an anchored mesh panel. In the first phase, the effect of a shift of the punching element along 
the central axes of the mesh panel is analysed. Successively, the punching element is "freely" moved on the mesh panel (a dense regular grid spaced by $4 \mathrm{~cm}$ is considered) in order to characterize the value of $F_{\max }$ and $\delta_{\max }$ with reference to a generic shift. The other parameters are the same as those in the reference test reported in Table 1.

In Fig. 19, the force-displacement curves obtained shifting the punching element along the panel central axes are reported. The same shifts are applied along both axes, namely $e_{j}= \pm l_{j} / 4$ and $e_{j}= \pm l_{j} / 2$ (subscript $j$ represents the shift direction). The results relating to a shift along the $x$-axis (i.e. $e_{x} \neq 0$ ) are reported in Fig. 19a, while the results relating to a shift along the $y$-axis (i.e. $e_{y} \neq 0$ ) are reported in Fig. 19b. In the former case, a significant variation of the mechanical response is observed both in terms of maximum sustainable force and out-of-plane stiffness of the $F-\delta$ curve. Furthermore, a non-monotonic effect on the $F-\delta$ curve is observed: for $e_{x}= \pm l_{x} / 4$ a decrease in the maximum force is observed $\left(F_{\max } \approx 0.85 F_{a}^{*}\right)$, while for $e_{x}= \pm l_{x} / 2$ the maximum force is greater than the one obtained from a centred case $\left(F_{\max } \approx 1.25 F_{a}^{*}\right)$. The shift $e_{x}= \pm l_{x} / 2$ also determines a significant increase in the stiffness of the mesh panel response (i.e. $\left.k_{75}\left(e_{x}= \pm l_{x} / 2, e_{y}=0\right) \approx 1.6 k_{75}^{*}\right)$. Conversely, a shift along the $y$-axis has a very low influence on the mesh panel response, and the $F-\delta$ curves obtained are almost coincident, as shown in Fig. 19b.

The effect of a generic shift $\left(e_{x}, e_{y}\right)$, on the maximum sustainable force and on the deflection value at failure, can
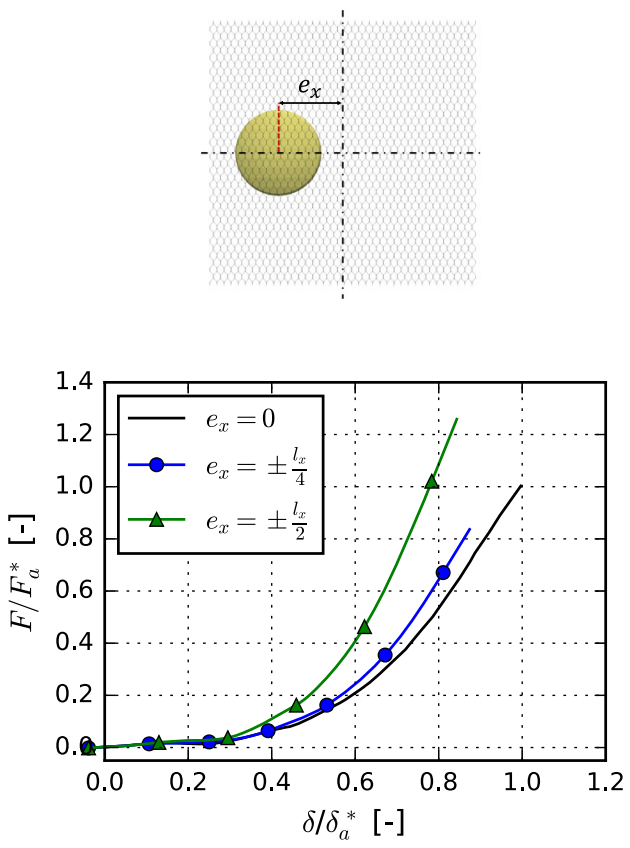

(a) be observed in the contour plots of Fig. 20a, b, respectively. They are obtained by a linear interpolation of the numerical data on the same grid used for defining the punch shift. In these plots, a value of generic coordinates $(x, y)$ refers to a simulation in which the punching element is centred in the same $(x, y)$ position. The light grey areas at the corners represent the zones where the punching element cannot be placed because of interference with the anchor plates (the fixed nodal particles in the model), while the latter are represented by the smaller black areas. The contour plots show that the effect of the position of the punching element, on the mechanical behaviour of the mesh, is not trivial. The anisotropy of the mesh mechanical response is particularly evident observing the trend of the deflection at failure. A very slight influence is observed moving the punching element in a vertical direction, while a shift along the horizontal direction entails a significant variation of $\delta_{\max }$. In particular, in the latter case, the deflection at failure diminishes when moving towards the boundary of the mesh panel. Concerning the trend of the maximum force $F_{\max }$, it is useful to consider the central part of the panel, namely $0.5 \mathrm{~m} \leq x \leq 2.5 \mathrm{~m}$ and $0.5 \mathrm{~m} \leq y \leq 2.5 \mathrm{~m}$, separately from the remaining four parts, which are comprised between the anchor plates. In the central part, shifting the punch along the $y$-direction leads to a slight variation of the $F_{\max }$, while the effect on $F_{\max }$ is more significant moving it along the $x$-direction. Three zones of local maximum of $F_{\max }$ are observable: they
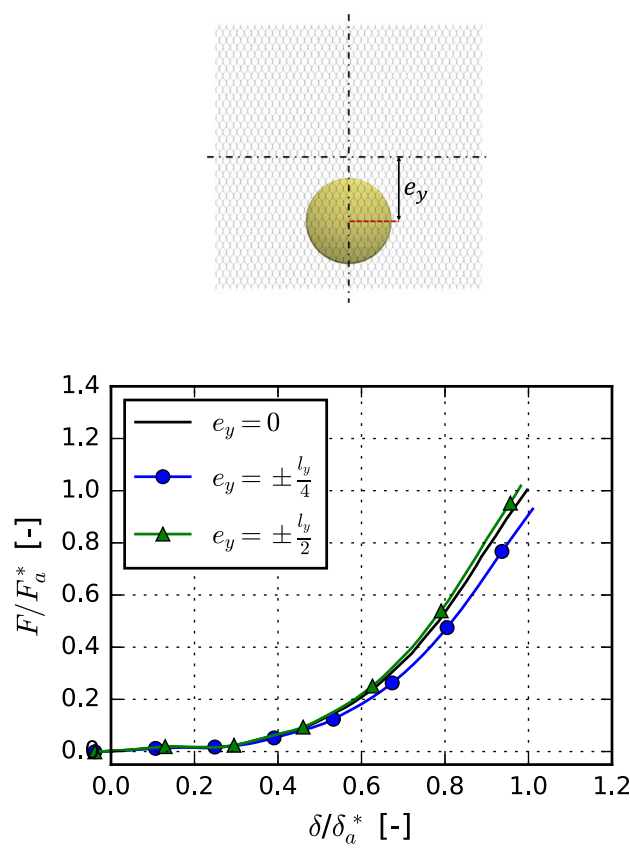

(b)

Fig. 19 Effect of the punching element position on the mechanical behaviour of the mesh panel for a shift along $\mathbf{a}$ the $x$-axis and $\mathbf{b}$ the $y$-axis in terms of force-displacement curve 


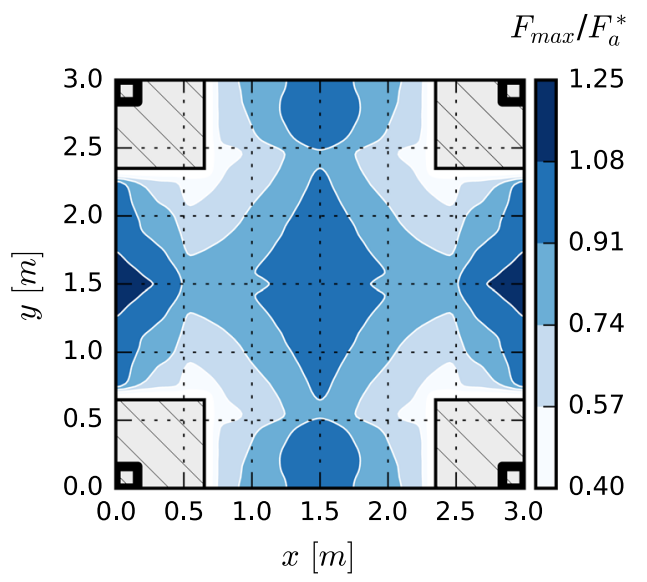

(a)

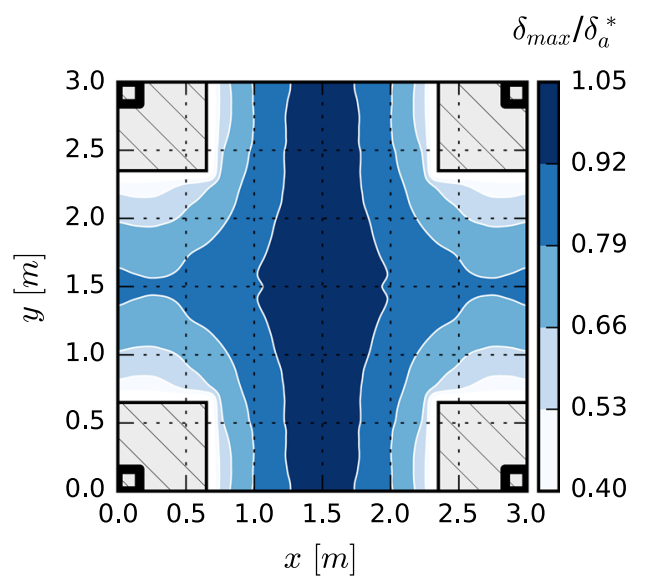

(b)

Fig. 20 Effect of the punching element position: a contour of force values at failure $F_{\max }$ and $\mathbf{b}$ contour of deflection values at failure $\delta_{\max }$. The contour plots are obtained from a linear interpolation of the numerical data on a regular grid with a spacing of $4 \mathrm{~cm}$

are approximately located corresponding to a symmetric position of the punching element with reference to the anchor plates, namely $(1.5 \mathrm{~m}, 1.5 \mathrm{~m}),\left(1.5 \mathrm{~m} \pm \frac{l_{x}}{2}, 1.5 \mathrm{~m}\right)$ and $\left(1.5 \mathrm{~m}, 1.5 \mathrm{~m} \pm \frac{l_{y}}{2}\right)$. The global maximum of $F_{\max }$ is observed for a punching element aligned in-between two anchor rows $\left(1.5 \mathrm{~m} \pm \frac{l_{x}}{2}, 1.5 \mathrm{~m}\right)$; furthermore this position also presents the highest overall mesh stiffness $\left(k_{75}\left(1.5 \mathrm{~m} \pm \frac{l_{x}}{2}, 1.5 \mathrm{~m}\right) \approx 1.6 k_{75}^{*}\right)$.

From this analysis, it can be argued that the relative position between the punching element and a possible unstable block influences the mechanical behaviour of the mesh panel. In particular, a significant gain in terms of both maximum sustainable force and out-of-plane stiffness of the mesh panel is observed when the block is comprised inbetween two anchor rows. It is therefore advisable, when possible, to consider the position of the unstable blocks on the slope face during the design and the installation phases of a secured drapery system in order to optimize its mechanical behaviour in terms of retaining capacity and panel deformability.

\subsection{Effect of the anchor plate size}

The dimension of the anchor plate is one of the controllable design parameters of a secured drapery system together with the mesh type, the anchor spacing and the panel aspect ratio (the latter two will be analysed in Sect. 4.4 and Sect. 4.5, respectively). In Sect. 4.1, it was observed that the maximum force sustainable by an anchored mesh panel is limited by the available resistance at the mesh-anchor connection and that the latter is mainly controlled by the anchor plate dimension (for the same mesh type). Nevertheless, a quantitative estimation of the effect of a variation of the dimension $d_{p}$ of the anchor plate was not provided.
In order to characterize a relationship between the maximum sustainable force $F_{\max }$ and the plate dimension, the latter is varied in the range $0.16 \mathrm{~m} \leq d_{p} \leq 0.64 \mathrm{~m}$ with an incremental step of $0.08 \mathrm{~m}$. The other parameters are the same as those in the reference test reported in Table 1. It is worth mentioning that in practical applications the anchor plate size is generally limited to $0.16 \mathrm{~m}<d_{p}<0.40 \mathrm{~m}$; however, larger values are adopted in the numerical model to obtain a wider range of values for the definition of a relationship between $F_{\max }$ and $d_{p}$.

The numerical results are reported in Fig. 21 in terms of force-displacement curve, while the trends of the maximum force and of the deflection at failure are shown in Fig. 22a, b, respectively. Considering the $F_{\max }$ value, a linear increasing trend is observed when increasing the plate dimension $d_{p}$. In fact, an increase in $d_{p}$ leads to a higher number of wires intercepted by the anchor plate, thus a higher force can be sustained by the mesh panel. It is also interesting to note that the linear trend is in analogy to what is observed when increasing the punching element

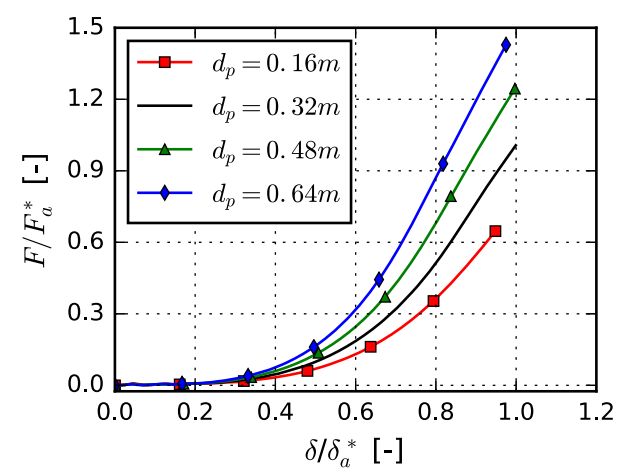

Fig. 21 Effect of the anchor plate size in terms of force-displacement curve 


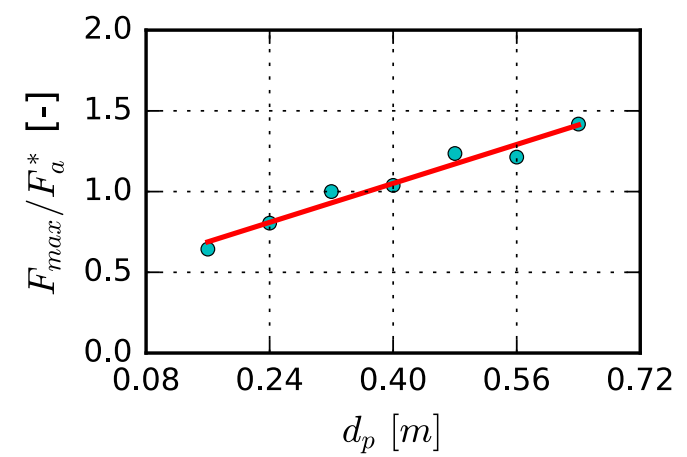

(a)

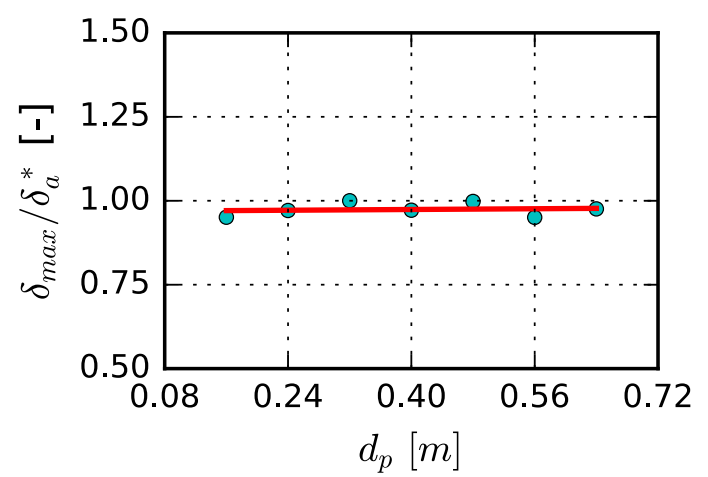

(b)

Fig. 22 Effect of the anchor plate size in terms of: a force $F_{\max }$ (fit Eq. 8) and b deflection $\delta_{\max }$ (fit Eq. 9) values at failure

size; in fact in this case the failure of the mesh is characterized by the "anchor-punching" mechanism $\left(d_{p}<D\right)$, and so the anchor plate can be seen as the "punching element". Conversely, the deflection $\delta_{\max }$ is almost unaffected by a variation of the anchor plate dimension. Finally, an increment of the stiffness of the $F-\delta$ curve is observed when increasing the anchor plate dimension (see Fig. 21). The step-like trend observed in Fig. 22a, b may be related to the discrete nature of the mesh model. In the latter, in fact, an anchor plate embraces a finite number of nodal particles (i.e. constrained numerical wires), which is dependent on the mesh cell geometry. Finally, from the numerical results, the influence of $d_{p}\left(d_{p}^{*}=0.32 \mathrm{~m}\right)$ on the maximum sustainable force by the mesh panel (linear least squares fit) and on the deflection at failure (linear least squares fit) can be described by the following relationships:

$$
\begin{array}{rlrl}
F_{\max } & =\left(0.48 \frac{d_{p}}{d_{p}^{*}}+0.45\right) F_{a}^{*} & d_{p} \geq 0.16 \mathrm{~m} \\
\delta_{\max }=\left(0.02 \frac{d_{p}}{d_{p}^{*}}+0.98\right) \delta_{a}^{*} & d_{p} \geq 0.16 \mathrm{~m}
\end{array}
$$

The non-null value of the intercept in the linear fit is associated with the fact that $d_{p}=0 \mathrm{~m}$ is meaningless. This value is related to the minimum dimension of the anchor plate that can be realistically considered in the model (i.e. $\left.d_{p}=0.16 \mathrm{~m}\right)$.

\subsection{Effect of the anchor spacing}

The anchor spacing $i$, or equivalently for a square panel, the panel side length (i.e. $i=l_{x}=l_{y}$ ), can be controlled during the design phase of a secured drapery system. It can be argued that the spacing $i$ controls the out-of-plane deformability of the mesh panel (and thus $\delta_{\max }$ ); however, it is not trivial to give a quantitative estimation of the effect of $i$ on the mesh mechanical response without performing a specific analysis. In this perspective, the anchor spacing is varied ranging from $i=1.25 \mathrm{~m}$ to $i=4.5 \mathrm{~m}$ with an incremental step of $0.25 \mathrm{~m}$. The other parameters are the same of the reference test reported in Table 1. Initially, the force-displacement curves obtained for four different values of anchor spacing (i.e. $1.5 \mathrm{~m}, 2.0 \mathrm{~m}, 3.0 \mathrm{~m}, 4.0 \mathrm{~m}$ ) are compared in Fig. 23. The numerical results confirm the assumption of an increment of panel deformability when increasing the anchor spacing as well as of the maximum deflection at failure.

In order to have a more precise overview of the effect of the anchor spacing on the mesh mechanical response, the trends of $F_{\max }$ and $\delta_{\max }$ as a function of $i$ are reported in Fig. 24a, b, respectively. The effect of a variation of the anchor spacing $i\left(i^{*}=3.0 \mathrm{~m}\right)$ on $F_{\max }$ can be estimated by the following relationship (linear least squares fit):

$$
F_{\max }=\left(0.16 \frac{i}{i^{*}}+0.84\right) F_{a}^{*} \text {. }
$$

A variation of $i$ seems to have a slight effect on the maximum force sustainable by the panel. The small increase in $F_{\max }$ observed when increasing $i$ may be related to a better redistribution of stresses for larger panels. The effect on the deflection at failure is instead significant, and a linear increment of the value of $\delta_{\max }$ with $i\left(i^{*}=3 \mathrm{~m}\right)$ is observed

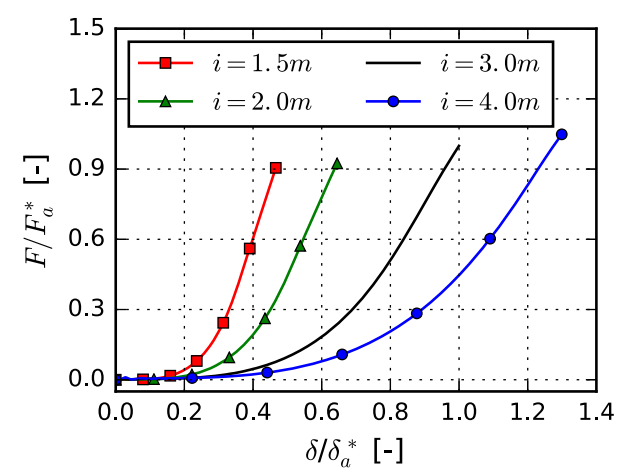

Fig. 23 Effect of the anchor spacing in terms of force-displacement curve 


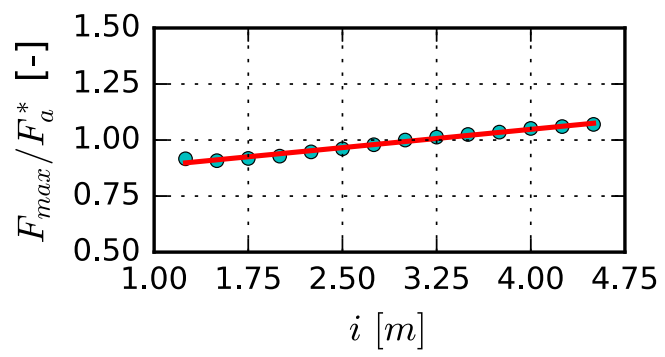

(a)

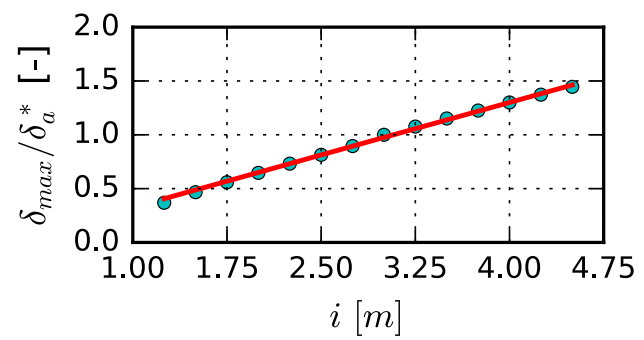

(b)

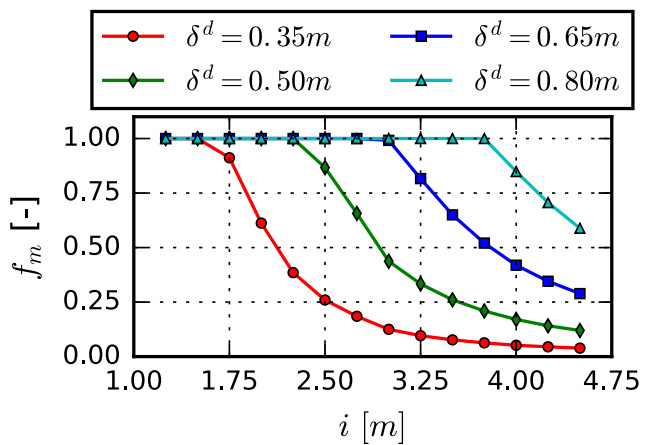

(c)

Fig. 24 Effect of the anchor spacing in terms of: a force $F_{\max }$ (fit Eq. 10) and $\mathbf{b}$ deflection $\delta_{\max }$ (fit Eq. 11) values at failure. c Evolution of the mobilized rate of the retaining capacity $f_{m}$ as a function of the anchor spacing

in Fig. 24b. The trend of $\delta_{\max }$ is well represented by the following relationship (linear least squares fit):

$\delta_{\text {max }}=0.98 \frac{i}{i^{*}} \delta_{a}^{*}$.

In light of the obtained results, for practical applications, the maximum force that a mesh panel can sustain may be assumed as independent from the anchor spacing. For instance, considering the two extreme values of $i$, namely $i=1.25 \mathrm{~m}$ and $i=4.5 \mathrm{~m}$, the increment in terms of $F_{\max }$ is approximately equal to $16 \%$. This increment is very limited, considering that the panel is more than three times larger in the second case. However, the role of the anchor spacing on the mesh panel performance, namely the relationship between the activated force for a given deflection of the panel, cannot be neglected.
In order to better understand this concept, let us define the force $F_{\delta}$ as the force value that can be mobilized for a panel deflection $\delta \leq \delta^{d}$, where $\delta^{d}$ represents the maximum admissible deflection in serviceability conditions. It should be noted that $\delta^{d}$ is here intended as a design parameter and, generally, does not coincide with the deflection $\delta$ experienced by the system. Therefore, if $\delta^{d} \leq \delta_{\max }^{i}\left(\delta_{\max }^{i}\right.$ is the maximum deflection related to a specific anchor spacing $i$ observed in the numerical characterization as reported in Fig. 24b), the system retaining capacity is only partially mobilized, namely $F_{\delta}<F_{\text {max }}^{i}$ ( $F_{\text {max }}^{i}$ is the maximum force related to a specific anchor spacing $i$ observed in the numerical characterization as reported in Fig. 24a). Conversely, if $\delta^{d} \geq \delta_{\max }^{i}$ then the system retaining capacity is fully mobilized even for a deflection $\delta<\delta^{d}$ and therefore one can consider $F_{\delta}=F_{\text {max }}^{i}$. Finally, the mobilized rate of the system retaining capacity is indicated as $f_{m}=F_{\delta} / F_{\max }^{i}$.

In Fig. 24c, the latter is reported as a function of the anchor spacing $i$, considering four different values of admissible deflection $\delta^{d}$. The $f_{m}-i$ curves thus defined permit evaluation of the system performance, quantifying the mobilized retaining capacity associated with a given limit condition on the maximum deflection of the system (i.e. $\delta \leq \delta^{d}$ ). They represent a useful design tool considering that limitations of system deformations, in serviceability conditions, are generally required, especially when secured drapery systems are applied along railways or other infrastructures. In fact, by means of the $f_{m}-i$ curves, it is possible to characterize the maximum anchor spacing that permits mobilization of the required rate of system retaining capacity, and at the same time fulfilling the condition $\delta \leq \delta^{d}$ on the system deformations.

For instance, if a limit deflection $\delta^{d}=0.50 \mathrm{~m}$ is imposed in serviceability conditions, the $f_{m}-i$ curve characterized by $\delta^{d}=0.50 \mathrm{~m}$ (green line with diamond marker in Fig. 24c) shows that the mesh panel retaining capacity is fully mobilized for $i \leq 2.25 \mathrm{~m}$ (i.e. $f_{m}=1.0$, with $\delta \leq \delta^{d}$ ), while the retaining efficiency of the intervention is very low (i.e. $f_{m}<0.5$, with $\delta=\delta^{d}$ ) for $i \geq 3.0 \mathrm{~m}$. Furthermore, considering that reduction of the anchor spacing entails a significant increment of the cost of the system, the benefit related to the use of $f_{m}-i$ curves also has an effect in financial terms.

\subsection{Effect of the mesh panel aspect ratio}

In order to extend the results related to a simple variation of the mesh panel dimension (i.e. the same variation of the anchor spacing along both directions presented in Sect. 4.4), the aspect ratio $A R=l_{x} / l_{y}$ is varied. The length 
of the panel sides is varied in the range $1.25 \mathrm{~m} \leq l_{j} \leq 4.5 \mathrm{~m}$ (incremental step of $0.25 \mathrm{~m}$ ) considering one side at a time. The other parameters are the same as those in the reference test reported in Table 1. Firstly, the effect of $A R$ on the force-displacement response of three equiareal panels (i.e.

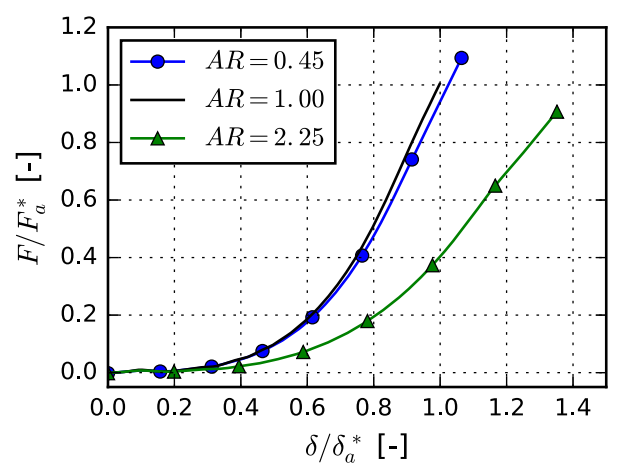

Fig. 25 Effect of the panel aspect ratio $\left(A R=l_{x} / l_{y}\right)$ in terms of forcedisplacement curve

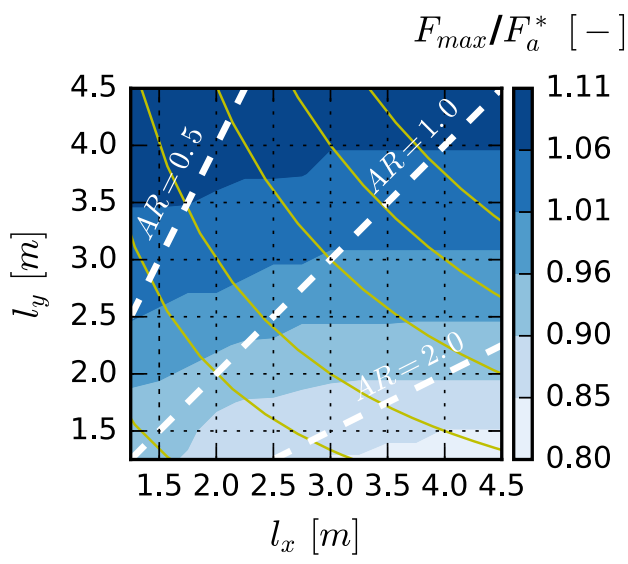

(a)
$2 \mathrm{~m} \times 4.5 \mathrm{~m}, 3 \mathrm{~m} \times 3 \mathrm{~m}, 4.5 \mathrm{~m} \times 2 \mathrm{~m}$ ) is considered. From the comparison of force-displacement curves reported in Fig. 25, it is evident that the adoption of a panel which is more elongated along the horizontal direction (i.e. $A R=2.25$ ), leads to a significant increment of panel deformability $\left(\delta_{\max } \approx 1.37 \delta_{a}^{*}\right)$ and also determines a reduction of the maximum sustainable force with respect to the square panel case $\left(F_{\max } \approx 0.91 F_{a}^{*}\right)$. Conversely, for $A R=0.45$, panel deformability is almost unaffected and an increase in the force sustainable by the mesh panel is observed $\left(F_{\max } \approx 1.11 F_{a}^{*}\right)$.

The effect of a generic variation of the panel aspect ratio on the mesh mechanical response can be observed in the contour plots of Fig. 26. The solid yellow lines indicate equiareal panels. The effect of a variation of $A R$ on $F_{\text {max }}$ is observable in Fig. 26a. It is evident that, for equiareal panels, an increment of $A R$ leads to a decrease in the maximum sustainable force by the mesh panel. This highlights the anisotropy that characterizes the mechanical

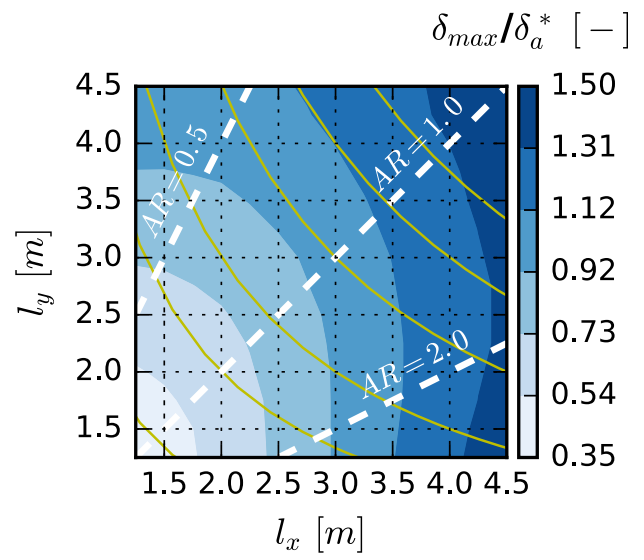

(b)

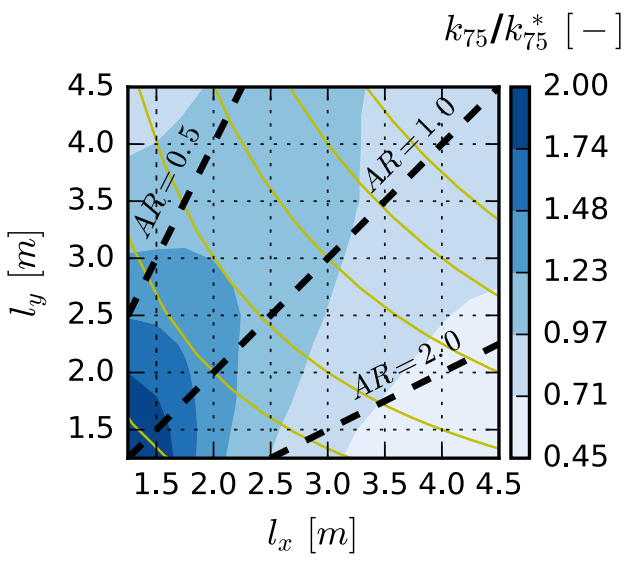

(c)

Fig. 26 Effect of the panel aspect ratio $\left(A R=l_{x} / l_{y}\right)$ in terms of: a force $F_{\max }, \mathbf{b}$ deflection $\delta_{\max }$ values at failure, $\mathbf{c}$ out-of-plane stiffness $k_{75}$. The plots are obtained from a linear interpolation of the numerical data and the solid yellow lines indicate equiareal panels (color figure online) 
behaviour of the hexagonal double-twisted wire mesh indicating that a square mesh panel may not be the optimal configuration. For a fixed value of $A R$ instead, increasing the panel size leads to an increase in the maximum sustainable force, which may be related to a better redistribution of the stress on larger mesh panels. This was also observed in Sect. 4.4 with reference to square panels. Considering a generic variation of $A R$, a reduction of the latter determines an increase in $F_{\max }$. Nevertheless, the effect on panel resistance seems to be controlled by the side length $l_{y}$ (side oriented with the double-twisted wire), a variation of $l_{x}$ having a negligible effect on $F_{\max }$. In fact, an increment of the side length $l_{y}$, while keeping constant $l_{x}$, permits a more efficient transmission of the force inside the mesh. The internal transmission mechanism is more and more oriented with the direction of the double-twisted wires; the mesh being characterized by better tensile resistance in such direction, this reflects in an enhancement of the mechanical response of the mesh in terms of sustainable force.

The effect of a variation of $A R$ on the deflection value at failure $\delta_{\max }$ is reported in Fig. 26b. Considering equiareal panels, $\delta_{\text {max }}$ increases when increasing $A R$; however, panel deformability seems to be more affected by a variation of the side length $l_{x}$ rather than a variation of the side length $l_{y}$. A general increment of $\delta_{\max }$ is also observed when increasing the dimension of the mesh panel (i.e. panel area) confirming the observation from Sect. 4.4.

Finally, the influence of the panel aspect ratio on the out-of-plane stiffness of the mesh panel is analysed. As before, this is quantified by using the stiffness parameter $k_{75}$. Observing the results reported in Fig. 26c, an increasing trend of $k_{75}$ is observed when decreasing the $A R$; however, the out-of-plane stiffness seems mostly controlled by the dimension of the mesh panel (i.e. panel area). It is interesting to note that the stiffness parameter $k_{75}$, for a given panel area, is greatest when the panel aspect ratio is in the range $0.65 \leq A R \leq 0.75$.

\subsection{Application example}

A calculation example is reported in the following in order to show the use of the analytical relationships presented in the previous paragraphs. The secured drapery system that is considered here is characterized by an anchor spacing $i$ of $2.5 \mathrm{~m}$ (a square anchor pattern is adopted, thus $l_{x}=l_{y}=2.5 \mathrm{~m}$ ) and the dimension of the anchor plates is equal to $d_{p}=0.25 \mathrm{~m}$. Finally, the nominal dimension of the unstable block considered is equal to $D=0.60 \mathrm{~m}$. The wire mesh is the hexagonal double-twisted mesh (nominal wire diameter of $2.7 \mathrm{~mm}$ ) described in Sect. 3.1. The characteristic values given by the manufacturer (obtained from an experimental characterization in UNI punch test conditions) are $F^{U}=73.3 \mathrm{kN}$ and $\delta^{U}=0.60 \mathrm{~m}$.

The analytical relationships derived from the numerical analysis, starting from the values obtained from the standard UNI laboratory characterization, can be applied in sequence in order to estimate the characteristic values to be used in the design phase of the mesh system $\left(F_{\max }=22.8 \mathrm{kN}\right.$ and $\left.\delta_{\max }=0.56 \mathrm{~m}\right)$. The procedure is summarized in Table 2 .

A simulation characterized by the same parameters considered in the calculation example (i.e. $i=l_{x}=l_{y}=2.5 \mathrm{~m}, d_{p}=0.25 \mathrm{~m}$ and $D=0.60 \mathrm{~m}$ ) was performed for an ex-post verification of the analytical relationships derived in the parametric analysis. The obtained values of the force and deflection values at failure are $F_{\max }=22.5 \mathrm{kN}$ and $\delta_{\max }=0.56 \mathrm{~m}$. The slight difference between these values and the ones obtained from the application of the analytical relationships confirms the validity of the latter. Moreover, the force-displacement curve (obtained from the simulation) permits the expected panel deflection to be estimated with reference to a given force value (the latter can be obtained from limit equilibrium analysis [28]) as schematically reported in Fig. 27.

Table 2 Application example of the analytical relationships $\left(i=2.5 \mathrm{~m}, d_{p}=0.25 \mathrm{~m}\right.$ and $\left.D=0.60 \mathrm{~m}\right)$

\begin{tabular}{lll}
\hline & $F_{\max }[\mathrm{kN}]$ & $\delta_{\max }[\mathrm{m}]$ \\
\hline UNI characteristic values & $73.3\left(F^{U}\right)$ & $0.60\left(\delta^{U}\right)$ \\
Field boundary conditions & $29.1\left(\alpha_{F} F^{U}\right)$ & $0.65\left(\alpha_{\delta} \delta^{U}\right)$ \\
Block dimension $D$ & 28.4 (Eq. 5) & 0.68 (Eq. 6) \\
Anchor plate dimension $d_{p}$ & 23.4 (Eq. 8) & 0.68 (unaffected) \\
Anchor spacing $i$ & 22.8 (Eq. 10) & 0.56 (Eq. 11) \\
Design characteristic values & 22.8 & 0.56 \\
\hline
\end{tabular}

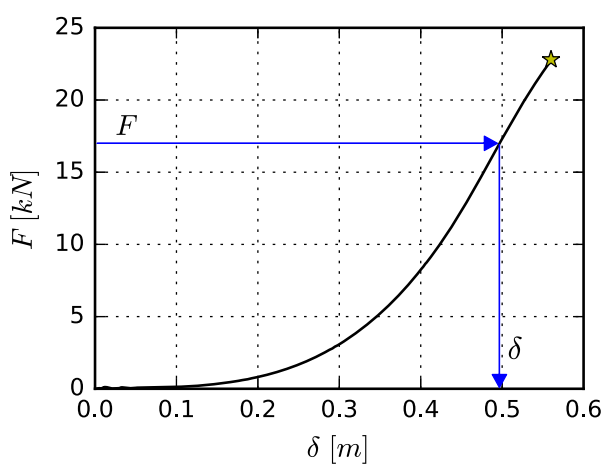

Fig. 27 Force-displacement response obtained from the numerical simulation (Test B1 configuration) of the mesh panel considered in the application example. The simulation is characterized by $l_{x}=l_{y}=2.5 \mathrm{~m}, d_{p}=0.25 \mathrm{~m}$ and $D=0.60 \mathrm{~m}$. The star indicates the couple $F_{\max }, \delta_{\max }$ obtained from the analytical relationships (see Table 2) 


\section{Conclusion}

In this study, the DEM was used to analyse the mechanical behaviour of a hexagonal double-twisted wire mesh panel subjected to a punching loading condition. The numerical mesh model was validated against experimental results of a standard UNI punch test. The DEM model had shown it was capable of efficiently reproducing the mechanical response of a wire mesh to an out-of-plane loading condition, requiring as input only the stress-strain relationships characterizing the tensile behaviour of the wire elements. Moreover, the model was able to correctly predict scale effects related to a variation of the dimension of the mesh panel and of the punching element.

In order to move towards a more realistic schematization of the field conditions, two large-scale test configurations, in which the mesh is locally anchored, were considered. A "periodic" loading condition (i.e. Test $B$ ) was shown to be more restrictive in terms of puncturing resistance and was therefore preferred. Finally, it was shown that a "periodic" single panel configuration (i.e. Test B1) is equivalent to the large-scale one in terms of mesh mechanical response, permitting a significant reduction of the computational time. The "periodic" single panel configuration was therefore adopted when following the analysis. It was shown that the mesh panel resistance provided by standard UNI punch test configuration overestimates the resistance of the wire mesh in field conditions. This is due to the difference in terms of boundary conditions between the experimental punch test and field applications. Subsequently, the mesh model was used to perform a parametric analysis aimed at quantifying the influence of the fundamental parameters characterizing the field conditions. The main results are summarized as follows:

- the size of the punching element controls the amount of the mesh-punch interacting surface, and determines the failure modality occurring in the mesh. Two different failure mechanisms were observed, namely a "block-punching" and an "anchor-punching" mechanism. The transition between the two is controlled by the available resistance at the mesh-plate connection, which is a function of the anchor plate dimension $d_{p}$ (for the same mesh type). The former mechanism occurs when the resistance at the mesh-block contact is lower than the mesh-plate connection resistance (a condition likely when the mesh is retaining a sharp block). In this case, a significant increment of the maximum sustainable force is observed when increasing the punching element size (Eq. 5 for $D \lesssim d_{p}$ ). When instead the failure is caused by the overcoming of the mesh-plate connection resistance (a condition likely when the mesh is retaining large and/or rounded blocks), the panel resistance is very slightly affected by a variation of the punching element size (Eq. 5 for $\left.D \gtrsim d_{p}\right)$;

- the position of the punching element influences the mechanical performance of the mesh panel. The effect of an eccentric load is strongly related to the anisotropy that characterizes the hexagonal double-twisted mesh; in particular, an eccentricity along the direction parallel to the double-twisted wires orientation has a negligible effect on the mesh response, while an eccentricity along the opposite direction strongly influences the mesh response. Three zones of local maximum for the sustainable force were observed, namely for a block centred on the mesh panel and when the block is inbetween two anchor plates. The global maximum is observed when the block is located in-between two anchor rows and vertically aligned with them. Concerning the panel's maximum deflection, this reduces when increasing the proximity between the retained block and the anchor plates in a direction orthogonal to the orientation of the double-twisted wires;

- the anchor plate dimension controls the maximum resistance of the mesh panel (i.e. mesh-plate connection resistance). Considering the case in which the mesh failure is characterized by the "anchor-punching" mechanism, the panel resistance linearly scales with the anchor plate dimension (Eq. 8). Instead, the maximum deflection of the panel, can be considered to be independent of the anchor plate dimension;

- the anchor spacing controls the deformability of the mesh panel. The maximum deflection linearly scales with the anchor spacing (Eq. 11), while the effect on the maximum force is very limited (Eq. 10). As the deformability of the mesh panel is ruled by the anchor spacing, the latter can be used in a performance perspective, namely to control the rate of the system retaining capacity associated with a certain deflection level of the mesh panel (e.g. maximum admitted deflection in serviceability conditions). This can be achieved by using the $f_{m}-i$ curves as defined in Sect. 4.4;

- the panel aspect ratio has a significant role in the panel mechanical response. In particular, considering equiareal panels, an enhancement of the mechanical behaviour was observed when considering panels that are more elongated along the double-twisted wires direction (i.e. $A R<1$ ). Moreover, it was shown that the side length $l_{y}$ has a significant effect on the puncturing resistance of the mesh panel, while the side length $l_{x}$ mostly influences panel deformability. Finally, the adoption of $0.65 \leq A R \leq 0.75$ seems to maximize the out-of-plane stiffness of the mesh panel. 
It is important to note that, in this work, the mesh panel is tested up to its failure. This permits the entire mechanical response (i.e. $F-\delta$ curve) to be provided and to define the ultimate limit condition of an anchored mesh panel. The analytical relationships presented in Sect. 4 permit extension of the mesh characteristic values provided by manufacturers (obtained from standard laboratory tests) to field conditions (i.e. anchored mesh panel) as reported in Sect. 4.6. Such information can be of great importance in practice, providing a more realistic mechanical characterization of mesh resistance in field conditions. Moreover, knowledge of the entire force-displacement behaviour permits estimation of the system deformations in serviceability conditions at the design phase. In this work, the hexagonal double-twisted wire mesh was considered; however, the extension of the approach used to other mesh types is straightforward. Further studies are ongoing in order to analyse the effect of a debris accumulation, which represents the other main cause of failure in secured drapery applications.

Acknowledgements This work was supported by the Research Grant awarded to A. Pol by the University of Padova. The authors would like to acknowledge the financial support of Maccaferri Innovation Center. The authors are grateful to Dr. Nicola Mazzon and Eng. Marco Deana for the fruitful discussions on the analyses performed. The authors are grateful to Dr. Klaus Thoeni for his invaluable advice. The authors also thank the two anonymous reviewers for their valuable and constructive comments.

Open Access This article is licensed under a Creative Commons Attribution 4.0 International License, which permits use, sharing, adaptation, distribution and reproduction in any medium or format, as long as you give appropriate credit to the original author(s) and the source, provide a link to the Creative Commons licence, and indicate if changes were made. The images or other third party material in this article are included in the article's Creative Commons licence, unless indicated otherwise in a credit line to the material. If material is not included in the article's Creative Commons licence and your intended use is not permitted by statutory regulation or exceeds the permitted use, you will need to obtain permission directly from the copyright holder. To view a copy of this licence, visit http://creativecommons. org/licenses/by/4.0/.

Funding Open access funding provided by Università degli Studi di Padova within the CRUI-CARE Agreement.

\section{References}

1. Albaba A (2015) Discrete element modeling of the impact of granular debris flows on rigid and flexible structures. Ph.D. thesis, Université Grenoble Alpes

2. Albaba A, Lambert S, Kneib F, Chareyre B, Nicot F (2017) DEM modeling of a flexible barrier impacted by a dry granular flow. Rock Mech Rock Eng 50(11):3029-3048. https://doi.org/10. 1007/s00603-017-1286-Z

3. Baek B, Karampinos E, Hadjigeorgiou J (2020) Understanding the impact of test configuration on welded-wire mesh laboratory test results. Rock Mech Rock Eng. https://doi.org/10.1007/ s00603-020-02198-w

4. Baraniak P, Stolz M, Schoevaerts B, Wendeler C (2016) New results of high-tensile steel meshes tested in first large scale field test application. In: Jacobsz (ed) Proceedings of the first Southern African geotechnical conference. Taylor \& Francis Group, pp 361-366

5. Bertolo P, Oggeri C, Peila D (2009) Full-scale testing of draped nets for rock fall protection. Can Geotech J 46(3):306-317. https://doi.org/10.1139/T08-126

6. Bertrand D, Nicot F, Gotteland P, Lambert S (2005) Modelling a geo-composite cell using discrete analysis. Comput Geotech 32(8):564-577. https://doi.org/10.1016/j.compgeo.2005.11.004

7. Bertrand D, Nicot F, Gotteland P, Lambert S (2008) Discrete element method (DEM) numerical modeling of double-twisted hexagonal mesh. Can Geotech J 45(8):1104-1117. https://doi.org/ 10.1139/T08-036

8. Bertrand D, Trad A, Limam A, Silvani C (2012) Full-scale dynamic analysis of an innovative rockfall fence under impact using the discrete element method: from the local scale to the structure scale. Rock Mech Rock Eng 45(August 2015):885-900. https://doi.org/10.1007/s00603-012-0222-5

9. Blanco-Fernandez E, Castro-Fresno D, Del Coz Díaz J, LopezQuijada L (2011) Flexible systems anchored to the ground for slope stabilisation: critical review of existing design methods. Eng Geol 122(3):129-145. https://doi.org/10.1016/j.enggeo.2011. 05.014

10. Bonati A, Galimberti V (2004) La valutazione sperimentale di sistemi di difesa attiva dalla caduta massi. In: Proceedings of: Bonifica dei versanti rocciosi per la protezione del territorio, pp 11-15. Trento

11. Bourrier F, Lambert S, Baroth J (2015) A reliability-based approach for the design of rockfall protection fences. Rock Mech Rock Eng 48:247-259. https://doi.org/10.1007/s00603-013-05402

12. Castro-Fresno D (2000) Estudio y análisis de las membranas flexibles como elemento de soporte para la estabilización de taludes y laderas de suelos y/o materiales sueltos. Ph.D. thesis, Universidad de Cantabria, Santander

13. Cazzani A, Mongioví L, Frenez T (2002) Dynamic finite element analysis of interceptive devices for falling rocks. Int J Rock Mech Min Sci 39(3):303-321. https://doi.org/10.1016/S13651609(02)00037-0

14. Coulibaly JB (2017) Modélisation numérique discrète du comportement mécanique sous impact des structures d'écrans de filets pare-pierres. Ph.d. thesis, Université Grenoble Alpes

15. Coulibaly JB, Ma Chanut, Lambert S, Nicot F (2017) Nonlinear discrete mechanical model of steel rings. J Eng Mech. https://doi. org/10.1061/(ASCE)EM.1943-7889.0001303

16. Cundall PA, Strack ODL (1979) A discrete numerical model for granular assemblies. Géotechnique 29(1):47-65. https://doi.org/ 10.1680/geot.1979.29.1.47

17. Effeindzourou A, Thoeni K, Giacomini A, Wendeler C (2017) Efficient discrete modelling of composite structures for rockfall protection. Comput Geotech 87:99-114. https://doi.org/10.1016/j. compgeo.2017.02.005

18. Escallón J, Boetticher V, Wendeler C, Chatzi E, Bartelt P (2015) Mechanics of chain-link wire nets with loose connections. Eng Struct 101:68-87. https://doi.org/10.1016/j.engstruct.2015.07.005

19. Escallón J, Wendeler C, Chatzi E, Bartelt P (2014) Parameter identification of rockfall protection barrier components through an inverse formulation. Eng Struct 77:1-16. https://doi.org/10. 1016/j.engstruct.2014.07.019

20. Gabrieli F, Pol A, Mazzon N, Deana ML (2020) Discrete element simulations of punch tests for the mechanical characterization of cortical meshes. In: da Fontoura SA, Jose Rocca R, Mendoza JP 
(eds) Rock mechanics for natural resources and infrastructure development. CRC Press, London, pp 3401-3407

21. Gabrieli F, Pol A, Thoeni K (2017) Comparison of two DEM strategies for modelling cortical meshes. Proceedings of particlebased methods-fundamentals and applications, particles 2017, pp 489-496

22. Gabrieli F, Pol A, Thoeni K, Mazzon N (2018) Particle-based modelling of cortical meshes for soil retaining applications. Numerical Methods in Geotechnical Engineering IX. CRC Press, London, pp 391-397

23. Gentilini C, Govoni L, de Miranda S, Gottardi G, Ubertini F (2012) Three-dimensional numerical modelling of falling rock protection barriers. Comput Geotech 44:58-72. https://doi.org/10. 1016/j.compgeo.2012.03.011

24. Gobbin L, Giacchetti G, Grimod A (2017) Results of tests to evaluate the tensile strength and the load bearing capacity of rockfall nets according to new ISO standards. In: 68th Highway geology symposium, pp 1-12. Marietta, Georgia

25. Govoni L, de Miranda S, Gentilini C, Gottardi G, Ubertini F (2011) Modelling of falling rock protection barriers. Int J Phys Model Geotech 11(4):126-137. https://doi.org/10.1680/ijpmg. 2011.11.4.126

26. Grassl H, Volkwein A, Anderheggen E, Ammann WJ (2002) Steel-net rockfall protection- experimental and numerical simulation. In: WIT transactions on the built environment. Lisbon

27. Grimod A, Giacchetti G (2003) Design approach for secured drapery systems. In: Sassa K, Canuti P, Yin Y (eds) Landslide science for a safer geoenvironment. Cham, pp 99-105

28. Grimod A, Giacchetti G (2014) Design approach for secured drapery systems. In: Sassa K, Canuti P, Yin Y (eds) Landslide science for a safer geoenvironment. Springer International Publishing, Cham, pp 99-105

29. Hearn G, Barrett RK, Henson HH (1995) Development of effective rockfall barriers. J Transp Eng 121(6):507-516

30. Karampinos E, Hadjigeorgiou J (2020) Quantifying the impact of bolting patterns on the performance of welded wire mesh. Geotech Geol Eng. https://doi.org/10.1007/s10706-020-01497-x

31. Li X, Zhao J (2018) A unified CFD-DEM approach for modeling of debris flow impacts on flexible barriers (May). https://doi.org/ 10.1002/nag.2806

32. Nicot F, Cambou B, Mazzoleni G (2001) Design of rockfall restraining nets from a discrete element modelling. Rock Mech Rock Eng 34(2):99-118. https://doi.org/10.1007/s006030170017
33. Nicot F, Cambou B, Mazzoleni G (2001) From a constitutive modelling of metallic rings to the design of rockfall restraining nets. Int J Numer Anal Meth Geomech 25(1):49-70

34. Pol A, Gabrieli F, Mazzon N (2020) Enhancement of design methodologies of anchored mesh systems using the discrete element method. In: Calvetti F, Cotecchia F, Galli A, Jommi C (eds) Geotechnical research for land protection and development. Springer International Publishing, Lecco, pp. 500-508. https:// doi.org/10.1007/978-3-030-21359-6_53

35. Pol A, Gabrieli F, Thoeni K, Mazzon N (2018) Discrete element modelling of a soil-mesh interaction problem. In: Litvinenko V (ed) Geomechanics and geodynamics of rock masses. CRC Press/ Balkema, Saint Petersburg, pp 877-882

36. Šmilauer V, Catalano E, Chareyre B, Dorofeenko S, Duriez J, Gladky A, Kozicki J, Modenese C, Scholtès L, Sibille L, Stránský J, Thoeni K (2015) Yade documentation. In: Release 2015-0309.git-a2be717. https://doi.org/10.1111/j.1440-1681.2007.04618.

37. Thoeni K, Giacomini A, Lambert C, Sloan SW, Carter JP (2014) A 3D discrete element modelling approach for rockfall analysis with drapery systems. Int J Rock Mech Min Sci 68:107-119. https://doi.org/10.1016/j.ijrmms.2014.02.008

38. Thoeni K, Lambert C, Giacomini A, Sloan SW (2013) Discrete modelling of hexagonal wire meshes with a stochastically distorted contact model. Comput Geotech 49(June 2014):158-169. https://doi.org/10.1016/j.compgeo.2012.10.014

39. UNI 11437: Rockfall protective measures-tests on meshes for slopes coverage (2012)

40. Volkwein A (2005) Numerical simulation of flexible rockfall protection systems. In: Computing in civil engineering, pp 1-11. Lisbon

41. Xu C, Tannant DD, Zheng W (2019) Discrete element analysis of the influence of bolt pattern and spacing on the force-displacement response of bolted steel mesh. Int J Numer Anal Methods Geomech. https://doi.org/10.1002/nag.2974

42. Xu C, Tannant DD, Zheng W (2020) Liu K (2020) Discrete element method and support vector machine applied to the analysis of steel mesh pinned by rockbolts. Int J Rock Mech Min Sci 125(June 2019):104163. https://doi.org/10.1016/j.ijrmms. 2019.104163

Publisher's Note Springer Nature remains neutral with regard to jurisdictional claims in published maps and institutional affiliations. 